\title{
Die Auslegung von familiengesellschaftsbezogenen Rechtsgeschäften
}

\author{
Prof. Dr. Jens Prütting, LL.M.oec., Hamburg* \\ und \\ Paul Schirrmacher, Hamburg*
}

Inhaltsübersicht

ZGR 2017, 829-864

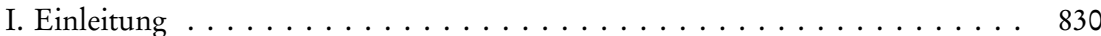

II. Grundsätzliche Vorbemerkungen zum Familienunternehmen und dem Kreis relevanter Rechtsgeschäfte . . . . . . . . . . . . . . . . . . .

1. Das „Familienunternehmen“ oder die „Familiengesellschaft“ als vage Anschauungsobjekte. . . . . . . . . . . . . . . . 832

2. System, Dynamik und Vielschichtigkeit des Familienunternehmens . . . . . 833

3. Familiengesellschaftsbezug als Auslegungsdeterminante . . . . . . . . 835

III. Auslegung als Einfallstor für die rechtliche Berücksichtigung der normativen Vielschichtigkeit in Familiengesellschaften . . . . . . . . . . . . . . . . . . . 835

1. Auslegungstatbestand und Auslegung . . . . . . . . . . . . . . . . . . . 835

2. Gegenständlicher Umfang des Auslegungstatbestands als Kernfrage . . . . . 836

IV. Der Vorrang der Auslegung vor (sonstigen) Kontrollmechanismen . . . . . . . 841

1. Überblick über die wesentlichen Kontrollmechanismen . . . . . . . . . . . . . . 841

2. Die Nichtigkeitsanordnungen der $\$ \mathbb{S} 134,138$ BGB . . . . . . . . . . . . 842

3. Die Inhaltskontrolle des Gesellschaftsvertrages nach $\ 242$ BGB . . . . . . . 845

4. Treuepflicht . . . . . . . . . . . . . . . . . . . . . 849

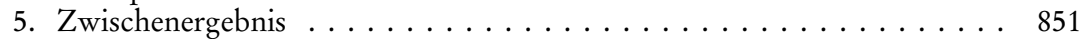

V. Spezifika des Gesellschaftsrechts vs. begleitende Vereinbarungen - zwingender Schutz durch Gesellschaftsrecht . . . . . . . . . . . . . . . . . . . 852

1. Akute Drittbetroffenheit (einheitliche vs. gespaltene Auslegung) . . . . . . . 852

2. Potentielle Drittbetroffenheit . . . . . . . . . . . . . . . . 861

VI. Zusammenfassung in Thesenform . . . . . . . . . . . . . . . . 864

* Prof. Dr. Jens Prütting, LL.M.oec. (Köln), Juniorprofessor an der Bucerius Law School, gestiftet von Gebr. Heinemann SE \& Co. KG, Direktor des Instituts für Medizinrecht und Habilitand am Lehrstuhl für Bürgerliches Recht, Handelsrecht, Internationales Privatrecht und Rechtsvergleichung der Universität Heidelberg (Prof. Dr. Marc-Philippe Weller) und geschäftsführender Direktor des Notarrechtlichen Zentrums Familienunternehmen der Bucerius Law School. PAul SCHIRrMacher ist wissenschaftlicher Mitarbeiter an der Bucerius Law School und Promovend am Institut von Prof. Prütting. 
Die besondere Dynamik von Familienunternehmen hat ihre Ursache in dem Einfluss der Unternehmerfamilie. Im Ausgangspunkt sind damit ökonomische und psychologische Realitäten angesprochen. Diese teilen sich jedoch auch der rechtlichen Sphäre mit, insbesondere mit Blick auf die das Familienunternehmen tragende Gesellschaft. In der Familiengesellschaft stehen sich nicht lediglich Minderbeiten und Mebrheiten, geschäftsführende und nicht-geschäftsführende Gesellschafter etc. wie in jeder anderen Gesellschaft gegenüber, sondern Individuen, die weit mebr als eine rational-ökonomische Beziehung verbindet (oder trennt). Damit gehen typischerweise auch rechtlich relevante Regelungsebenen neben dem Gesellschaftsvertrag einher, etwa in Gestalt von Gesellschaftervereinbarungen, Familienverfassungen usw. Der nachfolgende Beitrag gebt der Frage nach, ob und inwiefern die familiär bedingte Vielschichtigkeit von familienunternehmensbezogenen Regelungsebenen die Auslegung des Familiengesellschaftsvertrags beeinflusst.

The special dynamics of familiy businesses have their origin in the entrepreneurial family's influence. This primarily concerns economic and psychological realities. Those, however, relate to the legal sphere, in particular to the family company. Family companies, in contrast to any other company, do not only raise questions of majorities vs. minorities and managing vs. non-managing shareholders etc., but they comprise people united (or divided) in a relationship beyond rational economic categories. That usually entails legally relevant agreements in addition to the articles of association of the family company, e.g. shareholder agreements or family constitutions etc. This paper explores into whether and to what extent the family-driven multiplicity of agreements and provisions pertaining to the family business determines the interpretation of the articles of association of a family company.

\section{Einleitung}

Jean Giraudoux spottete einst „Die Phantasie trainiert man am besten durch juristische Studien. Nie hat ein Dichter die Natur so frei ausgelegt wie ein Jurist die Wirklichkeit. " ${ }^{1}$ Kritik dieser Art begegnet dem Juristen häufig und scheint auch vielfach ihre Berechtigung zu haben, werden doch getroffene Aussagen und sogar notariell beurkundete Absprachen ${ }^{2}$ später von Anwälten und Gerichten teilweise mit anderen Inhalten besetzt, als dies semantisch und grammatikalisch aus dem jeweiligen Text heraus erfasst werden könnte. Dies erscheint für manchen Beobachter willkürlich, was für den fachkundigen Juristen unmittelbar mit dem Arbeitsauftrag einhergeht, die geltende Rechtslage und deren Anwendung auf rechtsstaatliche Mängel hin zu überprüfen. Willkür ist - in den Grenzen akzeptabler Freiräume - nur den Rechtsunterworfenen im Umgang mit ihren Rechten und Rechtsgütern erlaubt, niemals jedoch dem Recht und jenen, die an seiner Umsetzung mitwirken. ${ }^{3}$ Die Auslegung von Willenserklärungen steht - neben dem korrekten Gesetzesverständnis - im Zentrum der Kritik. ${ }^{4}$ Mit ihr kann scheinbar je nach Einzelfall

1 Giraudoux, Der trojanische Krieg findet nicht statt, Paris 1935.

2 BGH NJW-Spezial 2017, 1.

$3 \mathrm{Zu}$ den verfassungsrechtlichen Hintergründen Kischel, in: BeckOK GG, Stand 15.8.2017, Art. 3 Rdn. $83 \mathrm{ff} \mathrm{m.w.} \mathrm{N.}$

4 Vgl. hierzu BGHZ 77, 301, 304; BGH NJW-RR 1990, 817, 818f; BGH NJW 1995, 1212, 1213. 
das gewünschte Ergebnis beliebig herbeigeführt werden, wodurch es ausschließlich auf die Weltsicht und Gerechtigkeitsvorstellungen der zur Entscheidung berufenen Stelle (Gericht, Schiedsgericht, Schlichtungsstelle etc.) ankäme. ${ }^{5}$ An diesem Punkt ist es die Aufgabe der Rechtswissenschaft, die Macht der Entscheider durch möglichst präzise Kriterien und Systeme zu begrenzen und den Betroffenen die Chance zu eröffnen, Judikate mit Sachargumenten zu hinterfragen und gebotenenfalls anzugreifen. Kann hierdurch die Verlässlichkeit und Vorhersehbarkeit von Entscheidungen auf ein akzeptables Maß gesteigert werden, werden Vertragsparteien aus der ex ante-Sicht in die Lage versetzt, ihrem Willen auch für den Fall späteren Streits im Wege der Regelbefolgung bei Erschaffung der Vertragsurkunde effektiv Ausdruck zu verleihen.

Ein für die Vertragspraxis von Familienunternehmen kritischer Bereich ist das korrekte Verständnis von Gesellschaftsverträgen. ${ }^{6}$ Es kostet die Rechtsprechung bereits einige Mühe, das im Vertragswerk geschriebene Wort im ursprünglichen Sinne der Parteien zutreffend zu interpretieren. ${ }^{7}$ Ein in seiner Funktionalität besonders schwieriges vertragliches Umfeld entsteht zudem regelmäßig bei familiengetragenen Gesellschaften, sofern in Bezug auf die konkrete Situation respektive die konkret in Rede stehende Gesellschaftsvertragsbestimmung des Einzelfalls gesellschaftsvertragsexterne Aspekte geeignet sind, das Vertragsverständnis wesentlich zu beeinflussen, zu verändern oder zu ergänzen. ${ }^{8}$ Das zentrale Anschauungsobjekt Gesellschaftsvertrag wird in diesen Fällen in ein vielschichtiges System unterschiedlicher Stakeholderinteressen eingebunden, die denselben über begleitende Gesellschaftervereinbarungen, Stammesstatute, Familiencharten oder gar gelebte Familientraditionen zu beeinflussen geeignet sein könnten. ${ }^{9}$ Dies muss zwangsläufig zu dem eingangs angesprochenen Problem fehlender Rechtssicherheit für die Rechtsunterworfenen führen. Ein möglichst scharf umrissenes System nachvollziehbarer und sachlich wohlbegründeter Auslegungskriterien stellt gerade in diesem Bereich keinen die Privatautonomie bedrohenden Selbstzweck zur richterlichen Machtverschaffung dar, sondern ist vielmehr geeignet, die bestehenden Unsicherheiten zu Gunsten der Ergründung des wahren Parteiwillens unter Berücksichtigung schutzwürdiger Verkehrsinteressen abzumildern. In diesem

5 Mit Erwägungen zu Gegenstand und gebotener Grenzziehung der ergänzenden Vertragsauslegung OECHSLER, Gerechtigkeit im modernen Austauschvertrag, 1997, S. 235 ff; SINGER, Selbstbestimmung und Verkehrsschutz im Recht der Willenserklärungen, 1995, S. $50 \mathrm{ff}$.

6 Instruktiv GRUNEWALD, ZGR 1995, $68 \mathrm{ff}$.

7 Vgl. BGH NJW 2015, 859.

8 Vgl. Prütting, in: Lange/Windthorst, Sicherung des Familieneinflusses in Familienunternehmen, 2017, S. $35 \mathrm{ff} \mathrm{m.w.N.}$

9 Näher Holler, Münchener Handbuch des Gesellschaftsrechts, Bd. 7, 5. Aufl., 2016, $\int 75$. 
Sinne einen Beitrag zur dogmatischen Erörterung und zur praktischen Handhabung zu leisten, ist Gegenstand und Ziel des nachfolgenden Beitrags.

\section{Grundsätzliche Vorbemerkungen zum Familienunternebmen und dem Kreis relevanter Rechtsgeschäfte}

\section{Das „Familienunternehmen" oder die „Familiengesellschaft" als vage Anschaunngsobjekte}

Es ist weithin umstritten, was unter einem Familienunternehmen zu verstehen ist. ${ }^{10}$ Kriterien wie eine bestimmte Quote der Anteilseignerschaft, die bei der Familie liegen müsse, ${ }^{11}$ mehrere aufeinanderfolgende Generationen ${ }^{12}$ oder die Einflechtung bestimmter Familienwerte ${ }^{13}$ sind nur ein Ausschnitt zahlreicher Erklärungsmuster. ${ }^{14} \mathrm{Im}$ Gesetz finden sich lediglich marginale Hinweise, jedoch keine für alle Bereiche belastbaren Vorgaben. So definiert $\ 1$ Abs. 1 Nr. 1 DrittelbG die „Familiengesellschaft“ als AG, deren Aktionär eine einzelne natürliche Person oder deren Aktionäre nach $\ 15$ Abs. 1 Nr. $2-8$ und Abs. 2 $\mathrm{AO}$ untereinander verwandt oder verschwägert sind. Derartige Gesellschaften unterliegen nicht dem Regime der unternehmerischen Mitbestimmung. Dass diese Umschreibung jedoch für das Gros aller sonstigen Abgrenzungsfragen zu eng sein dürfte, steht nach aktueller Forschung außer Frage. ${ }^{15}$ Letztlich bestimmt der Terminus des Familienunternehmens weniger eine trennscharfe Linie für konkrete Rechtsfragen denn vielmehr ein beachtliches Muster, aus welchem rechtlich relevante Vertrauensgesichtspunkte hervorgehen können, die sich gerade im Rahmen vorliegend interessierender Elemente der Einflussnahme wie der Auslegung des Gesellschaftsvertrages niederschlagen. ${ }^{16}$ Wenn ein Aspekt jedoch regelmäßig als notwendige, wenn auch nicht zwingend als hinreichende Voraussetzung anzuerkennen ist, so muss wohl ein beherrschender Einfluss der Unternehmerfamilie auf den Rechtsträger des Familienunternehmens gefordert werden. ${ }^{17}$ Im Übrigen existiert also der spezifische Typus

10 Eine Sammlung heranführender Überlegungen bietet Tänzler, Corporate Governance und Corporate Social Responsibility im deutschen Mittelstand, 2013, S. $9 \mathrm{ff}$, der mit Recht bereits die Reichweite des Begriffs Familie hinterfragt.

11 Vgl. Lange, BB 2005, 2585f; Uffmann, ZIP 2015, 2441, $2443 \mathrm{f}$.

12 Vgl. Churchill/Hatten, American Journal of Small Business, 11/3, 1987, S. $51 \mathrm{ff}$, die wenigstens eine Generationennachfolge verlangen.

13 Chua/Chrisman/Sharma, Entrepreneurship Theory \& Practice, 23/4, 1999, S. $19 \mathrm{ff}$.

14 Vgl. Uffmann, ZIP 2015, 2441, 2443 m. w. N.

15 In der einschlägigen Literatur findet sich keine Stimme, die besagte Definition zu einem allgemeinen Grundsatz erheben möchte.

16 Ausführlich Prütting, aaO (Fn. 8), S. 35 ff.

17 Näher Holler, aaO (Fn. 9), $\$ 75$ Rdn. 7 ff m.w.N. 
„Familienunternehmen“ nicht, jedoch gibt es Strukturmerkmale, Verhaltensweisen und Problemkonstellationen, die gerade in familiengetragenen Unternehmensstrukturen mit signifikanter Häufigkeit zu beobachten sind und an Hand derer eine Orientierung für die Rechtspraxis vielfach extrapoliert werden konnte.

\section{System, Dynamik und Vielschichtigkeit des Familienunternebmens}

Das Familienunternehmen zeichnet sich durch eine besondere Dynamik aus, die auf den Einfluss der Unternehmerfamilie zurückgeht. ${ }^{18}$ Diese Dynamik ist vielfach rechtlich kaum einzufangen und daher primär aus psychologischen und ökonomischen Gesichtspunkten von Interesse. ${ }^{19}$ Als eine gewisse Binsenweisheit kann vorangestellt werden: Es geht in Familienunternehmen um den Widerstreit von Geld, Macht und Liebe. ${ }^{20}$ Anders als in der klassisch unternehmerischen Situation der auf Gewinnerzielung angelegten (Publikums-)Gesellschaft, die ihre Belange und widerstreitenden Interessen ausschließlich auf gesellschaftsrechtlicher Ebene auszufechten hat und parallel zum Gesellschaftsvertrag allenfalls schuldrechtliche Gesellschaftervereinbarungen hinzutreten lässt, ist in der Familiengesellschaft mit dem innerfamiliären Kreis eine zweite Planungs- und Entscheidungsebene anzutreffen. ${ }^{21}$ Diese zweite Ebene verfolgt jedoch häufig nicht dieselben Ziele wie die unternehmenstragende Gesellschaft. Zentrale Aspekte seien im Folgenden kurz angeführt:

a. Die Familie zielt regelmäßig auf einen langfristigen Bestand des Unternehmens und möchte dieses entsprechend auch generationenübergreifend erhalten. ${ }^{22}$ Dabei drohen Interessenkollisionen mit Wünschen nach hohen Gewinnausschüttungen, umfangreicheren Abfindungsregelungen und Investoreninteressen, die nur kurz- oder mittelfristig Kapital anbieten. ${ }^{23}$ Zugleich entstehen erhebliche Probleme mit der Personalauswahl, da für die Leitung und Führung nicht selten primär Familienmitglieder erwogen werden. ${ }^{24}$

18 Vgl. Dauner-Lieb, in: Röthel, Verträge der Unternehmerfamilie, 2014, S. $181 \mathrm{ff}$.

19 Monographisch erarbeitet bei SIMON, Einführung in die Theorie des Familienunternehmens, 2012; PrYM, Die Bedeutung von Sozialkapital beim Internationalisierungsprozess von Familienunternehmen, 2013; TÄnzler, aaO (Fn. 10); Fraune, Nachfolge durch Fremdmanager, 2013; Siegfried, Trendentwicklung und strategische Ausrichtung von KMUs, 2015; RetTenmeIer, Stakeholder Support in Family Business, 2014.

20 Vgl. Koeberle-Schmid/Witt/Fahrion, in: Koeberle-Schmid/Witt/Fahrion, Family Business Governance, 2. Aufl., 2012, S. 41.

21 Vgl. Simon, aaO (Fn. 19), S. $81 \mathrm{ff}$ und Prym, aaO (Fn. 19), S. $283 \mathrm{ff}$.

22 Ausführlich mit rechtlicher Analyse Kalss/Ровst, Familienunternehmen, 2013, S. $697 \mathrm{ff}$. S. a. Hilti, FS Binz, 2014, S. $303 \mathrm{ff}$.

23 Vgl. Kalss/Pobst, aaO (Fn. 22), S. $489 \mathrm{ff}$.

24 Vgl. Langenscheidt, FS Binz, 2014, S. 393 ff; s. a. Fraune, aaO (Fn. 19), S. 4 ff. 
b. Soweit ein Familienunternehmen auf Fremdgeschäftsführung zurückgreift, um sich externen Sachverstand einzukaufen, ist das Fehlerpotential zu beobachten, den damit wie in anderen Unternehmen regelmäßig bestehenden Principal-Agent-Conflict nicht wahrzunehmen. ${ }^{25}$ Vielmehr wird häufig weiterhin so agiert, als gebe es weder das Problem adverser Selektion noch die Sorge auseinanderfallender Zielvorstellungen. ${ }^{26}$ Diese Problematik fällt häufig erst dann auf, wenn die Fremdgeschäftsführung schon zur Unzufriedenheit der Familie gestaltend eingegriffen hat.

c. Die im Unternehmen gemäß der Stimmberechtigung verteilte Macht reflektiert vielfach nicht die wahre Herrschaft, die zumeist in der Hand weniger Personen oder eines Familienpatriarchen liegt. ${ }^{27}$ Dabei existieren keineswegs immer schuldrechtliche Stimmbindungsverträge, sondern es ist häufig kraft familiärer Autorität eine tatsächlich beherrschende Stellung gegeben, die von den anderen Familienmitgliedern kaum angezweifelt wird, bis es zu einer ernstzunehmenden Auseinandersetzung kommt. ${ }^{28}$

d. Mit dem Vorgenannten im Zusammenhang steht das Problem, dass die Struktur und der Einfluss innerhalb der Unternehmerfamilie für Dritte nicht einsehbar sind. Auch bei Existenz einer Familienverfassung wird diese regelmäßig nicht öffentlich gemacht oder allenfalls als eine Businessversion zur Verfügung gestellt. Aber auch in jenen Fällen, in denen eine Bekanntmachung an die Gesellschaft und alle Gesellschafter erfolgt, ergeben sich vielfach ungeahnte Dynamiken durch die auch in der höchstrichterlichen Rechtsprechung schon teilweise anerkannte Ausstrahlungskraft auf den Gesellschaftsvertrag und die gesellschaftsrechtliche Treuepflicht. ${ }^{29}$

e. Ein besonderes Thema ist die Wertebindung im Familienunternehmen. Es wird an dieser Stelle sicherlich nicht geleugnet, dass auch ein familiengeführtes Unternehmen möglichst hohe Gewinne erwirtschaften will und dementsprechend auf Profiterzielung ausgerichtet ist. Zugleich finden sich jedoch andere Wertschöpfungsaspekte, die als teilweise danebenstehend und teilweise sogar übergeordnet von der Inhaberfamilie eingestuft werden. ${ }^{30}$ So geht es um den

25 Treffend hervorgehoben von MaY/Winter, Kein Erfolg ohne Vertrauen der Inhaber, FAZ vom 7.11.2016, Nr. 260, S. 18.

26 Näher zum Problemkreis Fraune, aaO (Fn. 19), S. $29 \mathrm{ff}$.

27 Statt vieler ECKert, Evolution von Familienunternehmen, 2008, S. $11 \mathrm{ff}$; KormanN, in: Scherer/Blanc/Kormann/Groth/Wimmer, Familienunternehmen, 2. Aufl., 2012, Kapitel 1 Rdn. 163.

28 Trefflich dargestellt bei den Unternehmergesprächen der Jahre 2011 - 2016 auf den Jahrestagungen des Notarrechtlichen Zentrums Familienunternehmen der Bucerius Law School. Eine belastbare statistische Erhebung hierzu existiert bislang - soweit ersichtlich - nicht.

29 Ausführlich PrÜtTING, aaO (Fn. 8), S. 35 ff.

30 Hierzu Ingelfinger, FS Binz, 2014, S. 318 ff; Zinkann, FS Binz, 2014, S. 854 ff. 
Familiennamen und wofür dieser steht, um eine besondere Verpflichtung für den Schutz der Beschäftigten und eine damit zusammenhängende Unternehmenskultur, starke Reinvestitionsimpulse durch den Wunsch nach Beständigkeit und Sicherheit sowie eine stark personelle Orientierung in Bezug auf Werte, für die die Familie schon seit Generationen einsteht und die - selbst wenn ihre Verfolgung überholt erscheint - um jeden Preis aufrechterhalten werden sollen. ${ }^{31}$

\section{Familiengesellschaftsbezug als Auslegungsdeterminante}

Durch die Brille des Rechtsrealisten betrachtet, zeigen die bisherigen Ausführungen, dass der Familiengesellschaftsbezug für alle Stakeholder der Familiengesellschaft ein wirtschaftlich zentrales Einflusskriterium bildet, dessen Macht sich im Recht widerspiegeln muss. Entscheidender und erster Ansatz - wie im Folgenden auch mit Blick auf die dogmatische Grundlage hin noch aufgezeigt wird - muss das bindende Rechtsgeschäftsverständnis aller Parteien sein. Dass es hierbei rechtlich relevante Einflusskriterien gibt, ist höchstrichterlich längst anerkannt. Die entscheidende und nicht hinreichend durchdrungene Frage ist, wie genau dieser rechtliche Einfluss wirkt und welche Grenzen ihm gesteckt sind.

\section{Auslegung als Einfallstor für die rechtliche Berücksichtigung der normativen Vielschichtigkeit in Familiengesellschaften}

\section{Auslegungstatbestand und Auslegung}

Die normative Vielschichtigkeit der Familiengesellschaft ${ }^{32}$ als praktischer Regelfall ist unbestreitbar und der Gedanke der rechtsgeschäftlichen Relevanz eben dieser Vielschichtigkeit in der Einzelbetrachtung der jeweils in Rede stehenden Schicht bestechend. Gleichwohl ist im Ausgangspunkt hervorzuheben, dass jene kennzeichnende Vielschichtigkeit keine eigenständige Auslegungsmethode oder gar Rechtsgeschäftslehre bedingen kann. Grund hierfür ist der bereits dargelegte Umstand, dass es den spezifischen Typus „Familienunternehmen“ nicht gibt - und damit auch keinen korrespondierenden Typus des Familiengesellschaftsvertrags. ${ }^{33}$ Überdies handelt es sich bei

31 Vgl. Gläsel, in: Koeberle-Schmid/Witt/Fahrion, Family Business Governance, 2. Aufl., 2012, S. 469 ff, Schween, in: Koeberle-Schmid/Witt/Fahrion, Family Business Governance, 2. Aufl., 2012, S. 473.

32 Mit umfangreichen weiteren Nachweisen Fleischer, ZIP 2016, $1509 \mathrm{ff}$ („Zwiebelschalenmodell“).

33 Vgl. oben II. 1. 
der Feststellung von Strukturmerkmalen, die für Familiengesellschaften charakteristisch sind, gerade um die einer treffenden Inhaltsbeschreibung eines Gesellschaftsvertrags vorgelagerte Ermittlung der für die Auslegung als Rechtserkenntnisprozess relevanten Tatsachen. Es geht also um den Auslegungsgegenstand. ${ }^{34}$ Dessen Ermittlung ist jedoch ebenfalls partiell Auslegungsaufgabe $e^{35}$ - und zwar auf der Grundlage der anerkannten Auslegungsmethoden.

Anders als bei der Auslegung bipolarer Rechtsgeschäfte ${ }^{36}$ wird sich im Vorfeld der Auslegung des Inhalts von Familiengesellschaftsverträgen typischerweise unproblematisch feststellen lassen, dass Willenserklärungen vorliegen. Diese werden entweder im Rahmen des Abschlusses des Gesellschaftsvertrags durch die Gründer ${ }^{37}$ oder des späteren originären oder derivativen Erwerbs der Mitgliedschaft abgegeben. Denn der Gesellschaftsvertrag erschöpft sich nicht im punktuellen kommunikativen Kontakt von Rechtssubjekten, sondern setzt eine verfasste Organisation ${ }^{38}$ ins Werk, wobei die tatsächliche Teilhabe an dieser Organisation - ungeachtet der Möglichkeit von Willensmängeln - regelmäßig keine ernstlichen Zweifel am rechtsgeschäftlichen Charakter damit einhergehender Erklärungstatbestände hervorrufen dürfte.

\section{Gegenständlicher Umfang des Auslegungstatbestands als Kernfrage}

Wesentlich kritischer ist hingegen die Frage nach der gegenständlichen Weite des Auslegungstatbestands, mithin der Regelungsebenen, welche für die Bestimmung der Bedeutung einer auslegungsbedürftigen Regelung, die auf einer der in Betracht kommenden Regelungsebenen verortet ist, zu berücksichtigen sind. Es ist folglich zu klären, ob die dargelegte - zunächst einmal faktische Vielschichtigkeit der normativen Ebenen auch eine ebenenübergreifende Auslegung gebietet, ob und inwiefern also der Ermittlung des Inhalts von Familiengesellschaftsstatuten rechtlich verbindlich eine Gesamtschau der weiteren möglicherweise erkenntnisleitenden vertragsexternen Umstände voranzugehen hat. Die Bestimmung dieses „Auslegungsstoffs“ ist wiederum selbst eine Auslegungsaufgabe, die Frage nach deren korrekter Umsetzung eine Rechts-,

34 Vgl. BGH NJW-RR 1992, 772, 773; Busche, Münchener Komm. z. BGB, 7. Aufl., 2015, \133 Rdn. 50f; Palandt/Ellenberger, Komm. z. BGB, 76. Aufl., 2017, $\mathbb{1 3 3}$ Rdn. 5; Staudinger/SINGER, Neubearb. 2017, $\mathbb{1 3 3}$ Rdn. 25.

35 Busche, aaO (Fn. 34), $\mathbb{1} 133$ BGB Rdn. 50f; vgl. auch Erman/Arnold, BGB, 15. Aufl., 2017, $₫ 133$ Rdn. 11.

36 Vgl. Busche, aaO (Fn. 34), $\$ 133$ BGB Rdn. 51 mit umfangreichen Rechtsprechungsnachweisen.

37 K. Sснміdт, Gesellschaftsrecht, 4. Aufl., 2002, \5 I 1 b), S. 76, \19 II 1 b), S. 551.

38 K. Schmidt, aaO (Fn. 37), \5 I 1 b), S. 77. 
keine Tatsachenfrage. ${ }^{39}$ Denn der Wortlaut markiert lediglich den Ausgangspunkt der Auslegung. ${ }^{40}$ Stellt der Wortlaut des Erklärten sich als unklar und mithin auslegungsbedürftig dar, sind in einem weiteren Schritt grundsätzlich auch die außerhalb des Erklärungsakts liegenden Begleitumstände in die Auslegung einzubeziehen, soweit sie einen Schluss auf den Sinngehalt der Erklärung zulassen. ${ }^{41}$ Dass es in Bezug auf Familiengesellschaften häufig Erklärungsquellen gibt, die erkenntnisleitend für die Bestimmung der Bedeutung des Gesellschaftsvertrags sein können, wurde bereits gezeigt. Im Kern geht es also darum, ob und wie sie tatsächlich auslegungsergebnisleitend wirken.

Diese Fragestellung wurde in der Literatur teilweise bereits erörtert, insbesondere für die rechtlichen Wirkungen von Familienverfassungen. ${ }^{42}$ Diese sollen für das Verständnis von Personengesellschaftsverträgen zur Auslegung herangezogen werden können, bei der Interpretation von Satzungen wegen des Grundsatzes der objektiven Auslegung ${ }^{43}$ hingegen nach bestrittener ${ }^{44}$ Meinung nicht. ${ }^{45} \mathrm{Im}$ Folgenden soll es jedoch zunächst nicht um die mittelbare Rechtswirkung schuldrechtlicher (wenn auch ggf. als Innen-GbR zu qualifizieren$\operatorname{der}^{46}$ ) Nebenvereinbarungen im Einzelfall gehen, sondern ob und inwiefern die Mehrschichtigkeit der Regelungsebenen generell einen auslegungsrelevanten Aspekt darstellt.

\section{a) Mebrschichtigkeit und Wechselwirkung von Regelungsebenen im Gesellschaftsrecht}

Die Mehrschichtigkeit von Regelungsebenen und deren Wechselwirkungen sind keine dem Gesellschaftsrecht fremde Herausforderung, wobei es jedoch an einer übergeordneten Dogmatik fehlt. Insbesondere bei Personengesellschaften kann sich der Inhalt des Gesellschaftsvertrags aus unterschiedlichen Quellen speisen: $\mathrm{Da}$ anders als bei der $\mathrm{GmbH}$ und bei der AG nicht gesetzlich

39 Vgl. BGH NJW 2003, 2235, 2236; BGH NJW 1995, 45, 46; BGH NJW 1992, 2817.

40 BGHZ 121, 13, 16; BGH NJW-RR 2007, 976; BGH NJW 1995, 1212.

41 BGH NJW-RR 2000, 1002, 1003; BGH NJW 2002, 1260, 1261; WendtLAND, in: Bamberger/Roth, BGB, 3. Aufl., 2012, \133 Rdn. 25; Palandt/Ellenberger, aaO (Fn. 34), \133 BGB Rdn. 15.

42 Prütting, aaO (Fn. 8), S. 35, 43 ff; Fleischer, ZIP 2016, 1509, 1517 f; Lange, in: Röthel/ K. Schmidt, Die Verträge der Familienunternehmer, 2013, S. 33, 43 f; Hueck, Die Familienverfassung - Rechtliche Konturen eines Instruments der Corporate Governance in Familienunternehmen, 2017, passim.

43 Dazu BGHZ 47, 172, 180; BGHZ 123, 347, 352; BGHZ 142, 116, 125.

44 Fleischer, ZIP 2016, 1509, 1518; Kalss, FS Binz, 2014, S. 343, 351; Glässer, FS Binz, 2014, S. 228, 235.

45 Wicke, Münchener Komm. z. GmbHG, 2. Aufl., 2015, $\ 3$ Rdn. 145 m.w. N.

46 Holler, aaO (Fn. 9), $\$ 75$ Rdn. 111. 
vorgeschrieben ist, dass der vollständige Wortlaut des Statuts stets in einer

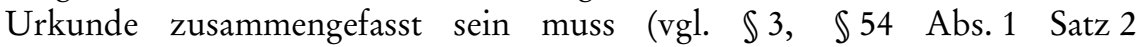

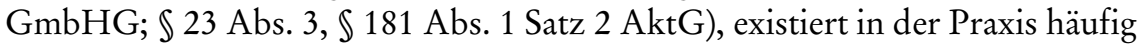
keine konsolidierte Fassung des Gesellschaftsvertrags, sondern lediglich eine Ausgangsfassung nebst einer Reihe nachfolgender Änderungsbeschlüsse, aus denen sodann nach dem Posterioritätsprinzip die jeweils aktuelle Fassung einer Regelung zu extrahieren ist. Die Zusammenführung und Ordnung der Regelungsebenen ist dabei eine in erster Linie logisch-textliche Aufgabe, keine Herausforderung auf der Rechtserkenntnisebene. Anders stellt sich die Situation dar, wenn zumindest prima facie lediglich faktisch für eine gewisse Dauer von geschriebenen Regelungen von Personengesellschaftsverträgen abgewichen wird. Dies wirft die Frage nach einer stillschweigenden Abänderung des Gesellschaftsvertrags auf, die nach allgemeinen vertragsrechtlichen Grundsätzen konstruktiv durchaus denkbar ist. ${ }^{47}$ Diesbezüglich ist genau jene für Familiengesellschaften charakteristische Mehrschichtigkeit zu konstatieren: Denn bei ständigen, von Gesellschaftsverträgen abweichenden Übungen selbst wenn deren Existenz als solche unstreitig ist - ist bereits im Ausgangspunkt unklar, ob sie als „Erklärungsquelle“ Teil des Auslegungsstoffs zur Ermittlung des Inhalts des Gesellschaftsvertrags oder ob sie schlicht faktische Abweichungen (Verstöße) gegen das gesellschaftsvertraglich Vereinbarte darstellen. So kann der Gesellschaftsvertrag Bestimmungen zur personell-fachlichen Qualifikation der Geschäftsführer enthalten, während die Familienverfassung insoweit ergänzende, modifizierende oder gar durchbrechende Bestimmungen enthält, die jedenfalls für Familienmitglieder gelebt werden, obgleich der Gesellschaftsvertrag keine qualitative Unterscheidung nach Familienzugehörigkeit kennt. Da entsprechende Klauseln in Personengesellschaftsverträgen keinem gesetzlichen Formerfordernis unterliegen, ist die Möglichkeit einer Vertragsänderung durch (von Rechtsbindungswillen getragene) externe Regelung oder durch Übung möglich. ${ }^{48}$

Deutlich komplexer stellt sich die gesellschaftsrechtliche Diskussion mit Blick auf Satzungen von Kapitalgesellschaften dar: Gemäß dem Trennungsprinzip ist zwischen Satzungsregelungen und wie auch immer gearteten anderen Regelungsebenen streng zu unterscheiden - und zwar bezüglich Inhalt und Rechtsfolgen. ${ }^{49}$ Ein Aspekt dessen ist der Grundsatz der objektiven Auslegung von Satzungen „aus sich selbst heraus“, d.h. unter Außerachtlassung

47 BGHZ 132, 263, 271; BGH NZG 2005, 625.

$48 \mathrm{Vgl}$. BGHZ 70, 331f, zur tatsächlichen Vermutung bei ständiger Übung, vgl. BGHZ 132, 263, 271. Siehe auch MaYer, Münchener Komm. z. GmbHG, 2. Aufl., 2015, $\$ 2$ Rdn. $150 \mathrm{ff}$ m.w. N.

49 Vgl. Wicke, aaO (Fn. 45), \$3 HGB Rdn. 139 ff; vgl. ferner Winter, ZHR 154 (1990), 259, $268 \mathrm{ff}$; Ulmer, NJW 1987, 1849, 1850 (beide in Bezug auf Gesellschaftervereinbarungen). 
sonstiger Vereinbarungen ${ }^{50}$, für dessen Richtigkeit insbesondere Formerfordernisse sowie drittschützende Aspekte angeführt werden. ${ }^{51}$ In der sog. „Kerbnägel“-Entscheidung ${ }^{52}$ hat der BGH allerdings einen GmbH-Gesellschafterbeschluss für anfechtbar erachtet, der zwar satzungs- und gesetzeskonform war, jedoch gegen eine zwischen allen Gesellschaftern getroffene außerstatutarische Abrede verstieß. Hier wurde die außerstatutarische Abrede also in ihrer Bedeutung als der Satzung letztlich vorrangig bewertet. ${ }^{53}$ Hingegen sollen satzungsdurchbrechende Beschlüsse (form-)unwirksam sein, wenn sie einen von der Satzung abweichenden Zustand begründen, d.h. nicht lediglich einen punktuellen Einzelfall in Abweichung von der Satzung regeln. ${ }^{54}$ Zwar ist die Umdeutung solcher Beschlüsse in schuldrechtliche Verpflichtungen $\mathrm{zu}$ entsprechendem Verhalten nicht ausgeschlossen, aber auch dann sollen sie nicht imstande sein, „eine bestimmte organisationsrechtliche Regelung der Satzung " zu ändern. ${ }^{55}$ Hervorzuheben ist in diesem Zusammenhang die Beschränkung auf organisationsrechtliche Regelungen, also korporative Regelungen, die nicht nur für alle oder einzelne der bei Inkrafttreten der in Rede stehenden Satzungsbestimmung vorhandenen Gesellschafter gelten, sondern für einen unbestimmten Personenkreis, zu dem sowohl gegenwärtige als auch künftige Gesellschafter und/oder Gläubiger der Gesellschaft gehö$\operatorname{ren}^{56}$ - in Abgrenzung zu schuldrechtlichen Bindungen, die lediglich in formeller Hinsicht Eingang in die Satzung gefunden haben. Nur korporative Satzungsbestimmungen sollen sich gegen andere Regelungsebenen durchsetzen und letztlich ohne Rücksicht auf jene anderen Regelungsebenen „aus sich heraus" auszulegen sein. ${ }^{57}$

\section{b) Familiengesellschaftsvertrag vs. Familiengesellschaftssatzung als Abgrenzungsmerkmal}

Die Trennlinie lautet nach h.M. folglich: Satzungen von Kapitalgesellschaften werden objektiv ausgelegt, wobei diese Auslegung vollständig revisibel ist. Personengesellschaftsverträge (mit Ausnahme der Publikumspersonengesell-

BGHZ 47, 172, 180; BGHZ 123, 347, 352; BGHZ 142, 116, 125.

Eingehend SCHOCKenHoff, ZGR 2013, 76, $87 \mathrm{ff}$.

52 BGH NJW 1983, 1910.

53 BGH NJW 1983, 1910: „so ist diese Regelung - auch ohne Bestandteil der Satzung zu sein - zumindest solange zugleich als eine solche der Gesellschaft zu behandeln“.

54 BGHZ 123, 15, 19; vgl. hierzu Scholz/Priester, Komm. z. GmbHG, 11. Aufl., 2015, \53 Rdn. 29 ff; LeUSCHNER, ZHR 180 (2016), 422, $426 \mathrm{f}$.

55 BGHZ 123, 15, 19; wortgleich jüngst: OLG Düsseldorf GmbHR 2017, 36, 37 m. Anm. PRIESTER.

56 Vgl. zum Begriff BGHZ 123, 347, 350.

57 BGHZ 14, 25, 36 f; BGHZ 123, 347, 350. 
schaften) hingegen sind nach den allgemeinen Grundsätzen der $\mathbb{S} 133,157$ BGB subjektiv auszulegen und nur ergänzend zu objektivieren ${ }^{58}$, wobei die Erforderlichkeit konkreter Feststellungen sowie der von jenen Feststellungen gegebenenfalls abhängigen konkreten Beweiswürdigung die Auslegung als solche dem Tatrichter zuweist und die Revisionsinstanz auf die Überprüfung der Rechtsfrage der korrekten Auslegungsmethode beschränkt. ${ }^{59}$ Konsequenz dieser Sichtweise ist ein fundamentaler Unterschied hinsichtlich der rechtlichen Berücksichtigungsfähigkeit ebenenübergreifender Regelungen zwischen Familienkapital- und Familienpersonengesellschaften.

Jedoch finden sich in der Rechtsprechung des BGH bemerkenswerte Ausnahmen: Denn einerseits wird in Bezug auf Kapitalgesellschaften konzediert, dass ,außerhalb der Satzung liegende Sachzusammenhänge [...] unter Umständen dann berücksichtigt werden [können], wenn deren Kenntnis bei den Mitgliedern und Organen allgemein vorausgesetzt werden kann “. ${ }^{60}$ Andererseits werden Gesellschaftsverträge von Publikumspersonengesellschaften objektiv, d.h. wie Satzungen von Kapitalgesellschaften, ausgelegt ${ }^{61} \mathrm{~K}$. Schmidt hat unter Verweis hierauf schon früh dargelegt, dass die kategorische Trennung zwischen Satzungen und Personengesellschaftsverträgen im Einzelfall von mangelhafter Überzeugungskraft sein $k^{2} n^{62}$ und jener Trennung eine rein materielle Betrachtung gegenübergestellt, die rechtsformunabhängig immer dann zu einer objektiven Auslegung führt, wenn die in Rede stehende Klausel einen „überindividuellen verbandsrechtlichen Charakter" ${ }^{\prime 63}$ aufweist. Darüber hinaus ist es nach $K$. Schmidt denkbar, dass verbandsintern ein vom verbandsexternen objektiven Verständnis abweichendes subjektives Verständnis des Gesellschaftsvertrags oder der Satzung verbindlich ist, wobei es nicht abstrakt bestimmbar sein soll, inwieweit auch Rechtsnachfolger an jenes subjektive Verständnis gebunden sind. ${ }^{64}$

Die beiden letztgenannten Gesichtspunkte - subjektives Verständnis und Rechtsnachfolge - sind mit Blick auf Familienunternehmen und die Vielschichtigkeit der darauf bezogenen Regelungsebenen von besonderer Brisanz ${ }^{65}$. Denn typischerweise werden Auseinandersetzungen über das „richtige“ - juristisch

58 BGH NZG 2014, 1296, 1299 m. w. N.

59 BGH, Urt. v. 29.9.1954 - II ZR 331/53, DB 1954, 882.

60 BGHZ 123, $15=$ DnotZ 1994, 313.

61 BGH NZG 2016, 424, 426; 2013, 738; 2012, 1345.

62 K. Schmidt, aаO (Fn. 37), \5 I 4 b), S. 89 f; K. Schmidt, Münchener Komm. z. HGB,

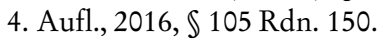

63 K. Schmidt, aaO (Fn. 37), \5 I 4 b), S. 90.

64 K. Sснмidt, aaO (Fn. 37), \5 I 4 c), S. 91.

65 Die besonders komplexe Fragestellung der „Wissensvererbung“ in Familienunternehmen wird in dieser Abhandlung nicht näher erörtert, sondern in einer eigenständigen, bereits ins Werk gesetzten Folgebetrachtung dezidiert analysiert. 
tragfähige - Verständnis von Satzungsklauseln gerade zwischen Gesellschaftern ausgetragen, die entweder Gründungsgesellschafter oder deren direkte oder indirekte Nachfolger von Todes wegen oder in vorweggenommener Erbfolge sind, sodass die Beschränkung der Möglichkeit einer subjektiven Auslegung auch materiell korporativer Satzungsbestandteile potenziell eine erhebliche Reichweite hat.

\section{Der Vorrang der Auslegung vor (sonstigen) Kontrollmechanismen}

Neben der vorab bereits grundlegend betrachteten Möglichkeit, die Statuten von Familiengesellschaften unter Berücksichtigung einer Familienverfassung oder anderer begleitender Vereinbarungen auszulegen, kommen bei einem zumindest scheinbaren - Widerspruch von Gesellschaftsvertrag und sonstigen normativen Ebenen - mit der Familienverfassung als Prototyp sonstiger Regelungsebenen - auch rechtsgeschäftsspezifische und gesellschaftsrechtsspezifische Kontrollmechanismen zur Auflösung bestehender Widersprüche in Betracht. Es stellt sich damit allem voran die Frage des Verhältnisses eben dieser zur Auslegung.

\section{1. Überblick über die wesentlichen Kontrollmechanismen}

Gedanklicher Ausgangspunkt ist stets der Gesellschaftsvertrag selbst und seine korrekte Interpretation als Grundlage einer jeden Gesellschaft. ${ }^{66}$ Eine privatrechtliche Gesellschaft ohne einen ihr zugrundeliegenden Gesellschaftsvertrag ist schlechthin nicht denkbar, da sie auf keine andere Weise - weder durch einen bloßen Hoheitsakt noch durch das „soziale Faktum ihres Vorhandenseins" - entstehen kann. ${ }^{67}$ Der Gesellschaftsvertrag ist dabei zugleich Schuldund Organisationsvertrag, ${ }^{68}$ sodass im Grundsatz auch die $\$ \mathbb{S} 104 \mathrm{ff}$. BGB zur Anwendung gelangen. ${ }^{69}$ Die schärfsten Kontrollschwerter des allgemeinen bürgerlichen Rechts sind in Bezug auf den Inhalt oder das Zustandekommen die $\mathbb{S} 134$ und 138 BGB. Ferner wird - zumindest für bestimmte Gesellschaftstypen - angenommen, dass deren Gesellschaftsverträge zusätzlich einer

66 Windbichler, Gesellschaftsrecht, 23. Aufl., 2013, \6 Rdn. 1; Grunewald, Gesellschaftsrecht, 9. Aufl., 2014, $\mathbb{1} 1$ Rdn. 2; so explizit für die oHG und die KG: K. Schmidt, aaO (Fn. 62), $₫ 105$ HGB Rdn. 27.

67 K. Schmidt, aaO (Fn. 37), $₫ 5$ I 1.

68 Für die Personengesellschaften: C. SCHÄFER, Gesellschaftsrecht, 2. Aufl., 2011, \5 Rdn. 11; für die Kapitalgesellschaften: Drygala/Staake/Szalai, Kapitalgesellschaftsrecht, 2012, $\ 4$ Rdn. 18.

69 Fastrich, in: Baumbach/Hueck, Komm. z. GmbHG, 21. Aufl., 2017, 22 Rdn. 5 f; SCHÄFER, in: Henssler/Strohn, Gesellschaftsrecht, 3. Aufl., 2016, \$2 GmbHG Rdn. 4 ff. 
Inhaltskontrolle unterzogen werden können, die an eine AGB-Kontrolle erinnert. Einen speziell für die AG vorgesehenen Fall der Inhaltskontrolle normiert zudem $\$ 23$ Abs. 5 Satz 1 AktG (Satzungsstrenge). ${ }^{70}$ Die Vertragsfreiheit der Aktionäre wird im Interesse der Gläubiger und zukünftiger Aktionäre begrenzt und die Aktie und damit die Gesellschafterstellung zu einem „standardisierten Produkt" stilisiert. ${ }^{71}$ Ein Verstoß gegen $\ 23$ Abs. 5 Satz 1 AktG führt zur Nichtigkeit. ${ }^{72}$ Demgemäß besteht im Aktienrecht für die Frage nach dem Einfluss außerstatutarischer Vereinbarungen im Vergleich zu den anderen Gesellschaftsformen der geringste Spielraum. ${ }^{73}$

Schließlich kommt aber auch die gesellschaftsrechtliche Treuepflicht als ein in Bezug auf Gesellschaftsverträge und der Ausübung von Gesellschafterrechten wichtiges Steuerungsinstrument in Betracht. ${ }^{74}$

Mithilfe des hier abstrakt skizzierten Konglomerats an Ansatzpunkten zur Kontrolle von gesellschaftsrechtlichen Rechtsgeschäften wird weitgehend sichergestellt, dass sich die in der Gesellschaft getroffenen Maßnahmen im Rahmen des gesetzlich und gesellschaftsvertraglich Zulässigen halten, mithin die Privatautonomie der Gesellschafter durch staatliche Vernunft begrenzt wird. $\mathrm{Ob}$ und inwieweit dies gutzuheißen ist, bleibt einer eigenständigen Erörterung vorbehalten.

\section{Die Nichtigkeitsanordnungen der $\iint 134,138 B G B$}

Die $\int \mathbb{S} 134,138$ BGB geben den gesetzlichen Rahmen vor, den ein Rechtsgeschäft mindestens einhalten muss und sind daher Bestandteil einer gesetzlichen "Inhaltskontrolle im weiteren Sinne“ ${ }^{75}$ Anhand der $\$ \mathbb{S} 134,138$ BGB werden nicht nur der Gesellschaftsvertrag, sondern auch sämtliche (gesell-

70 Vgl. Limmer, in: Spindler/Stilz, Komm. z. AktG, 3. Aufl., 2015, \$23 Rn 28f; VetTer, in: Henssler/Strohn, Gesellschaftsrecht, 3. Aufl., 2016, $\$ 23$ AktG Rdn. $22 \mathrm{ff}$.

71 Kосн, in: Hüffer/Koch, Komm. z. AktG, 12. Aufl., 2016, \$23 Rdn. 34.

72 Pentz, Münchener Komm. z. AktG, 4. Aufl., 2016, $\$ 23$ Rdn. 170 m.w. N. und Übersicht zur Gegenansicht, wonach sich die Rechtsfolgen eines Verstoßes gegen $\ 23$ Abs. 5 Satz 1 AktG aus $\$ 241$ Nr. 3 AktG ergeben.

73 Vgl. Fleischer, ZIP 2016, 1509; vgl. zu schuldrechtlichem Einfluss auf die korporative Sphäre der Aktiengesellschaft Bochmann, Covenants und die Verfassung der Aktiengesellschaft, 2012, S. $40 \mathrm{ff}$.

74 Verdeutlicht wird die Einordnung als Steuerungsinstrument insbesondere durch die mit den bei ihrer Verletzung verbundenen Rechtsfolgen, vgl. dazu: FAstrich, aaO (Fn. 69), $\$ 13 \mathrm{GmbHG}$ Rdn. 30.

75 Armbrüster, Münchener Komm. z. BGB, 7. Aufl., 2015, $\mathbb{1 3 4}$ Rdn. 1; Busche, Münchener Komm. z. BGB, 7. Aufl., 2015, vor $\$ 145$ Rdn. 25 ff. 
schaftsbezogenen) Rechtsgeschäfte und damit insb. auch (Gesellschafter-)Beschlüsse kontrolliert. ${ }^{76}$

Für die vorliegende Untersuchung hat $\ 134$ BGB jedoch keine Bedeutung, da Absprachen unterhalb des Gesetzesbegriffs i.S.d. Art. 2 EGBGB, wie dies für vertragliche und sonstige Vereinbarungen gilt, ${ }^{77}$ keinen Verbotsgesetzcharakter begründen.

Relevant ist dagegen die Nichtigkeitsanordnung des $₫ 138$ Abs. 1 BGB bei Verstoß gegen die "guten Sitten“. ${ }^{78}$ Nach h. M. sind unter dem Begriff der guten Sitten jedenfalls solche Verhaltensgebote zu verstehen, die sich aus der vorherrschenden Wertvorstellung und damit aus der Rechts- und Sozialmoral ergeben. ${ }^{79}$ Anders als $\$ 134$ BGB legt $\$ 138$ BGB damit gerade auch außerrechtliche Maßstäbe an. ${ }^{80}$ Die Sittenwidrigkeit kann sich dabei entweder aus dem Inhalt des Rechtsgeschäfts selbst (sog. Inhaltssittenwidrigkeit) oder aus den Umständen des Zustandekommens (sog. Umstandssittenwidrigkeit) ergeben. ${ }^{81}$ Auch an dieser Stelle hält das Aktienrecht zumindest mit Blick auf die Inhaltssittenwidrigkeit von Hauptversammlungsbeschlüssen mit $\mathbb{2 4 1} \mathrm{Nr} .4$ AktG eine Sonderregelung bereit. ${ }^{82}$

Zur Feststellung einer etwaigen, hier im Vergleich zur Inhaltssittenwidrigkeit relevanteren Umstandssittenwidrigkeit bedarf es einer Gesamtwürdigung der Umstände des Einzelfalles. ${ }^{83} \mathrm{Da}$ dabei auch äußere Einflüsse berücksichtigt werden, ${ }^{84}$ kann es jedenfalls nicht a priori ausgeschlossen sein, für die vorzunehmende Gesamtschau etwa eine Familienverfassung und das an ihr ausgerichtete langährige Verhalten der Gesellschafter heranzuziehen.

Nicht abschließend geklärt ist, in welchem Verhältnis $₫ 138$ Abs. 1 BGB zur Auslegung steht. Erkenntnisse dazu können aber etwa der Rechtsprechung zu den sog. Buchwertklauseln entnommen werden. Buchwertklauseln können wegen einer mit Ihnen einhergehenden Knebelung des Gesellschafters gegen

76 Für $\ 134$ BGB: BGHZ 59, 369, 372; Armbrüster, aaO (Fn. 75), $\ 134$ BGB Rdn. 23; DÖRNER, in: HK-BGB, 9. Aufl., 2017, $\ 134$ Rdn. 2; für $\ 138$ BGB: BGHZ 108, 21, 27; DÖRnER, aaO, $\int 138$ BGB Rdn. 2; Ulmer, NJW 1979, 81, 82 m.w. N.

77 Mansel, in: Jauernig, Komm. z. BGB, 16. Aufl., 2015, $\mathbb{1} 134$ Rdn. 8; Dörner, aaO (Fn. 76), $\$ 134$ BGB Rdn. 3.

78 Zur Ausfüllungsbedürftigkeit dieses unbestimmten Rechtsbegriffs ARmbrüster, aaO (Fn. 75), $₫ 138$ BGB Rdn. 11.

79 Wendtland, in: BeckOK BGB, Stand 19.6.2017, \138 Rdn. 16.

80 Looschelders, in: NK-BGB, 2. Aufl., 2011, $\$ 138$ Rdn. 34.

81 Armbrüster, aaO (Fn. 75), $\ 138$ BGB Rdn. 9; Dörner, aaO (Fn. 76), $\ 138$ BGB Rdn. 4.

82 Armbrüster, aaO (Fn. 75), $\ 138$ BGB Rdn. 9.

83 Armbrüster, aaO (Fn. 75), $\$ 138$ BGB Rdn. 8.

84 BGHZ 107, 92, 97; WendtLand, aaO (Fn. 79), \138 BGB Rdn. 21. 
die guten Sitten verstoßen, wenn sie schon im Zeitpunkt des Vertragsabschlusses dazu führen, dass das Kündigungsrecht eines Gesellschafters wegen einer erheblichen Differenz von vertraglichem Abfindungsanspruch und wirklichem Wert in tatsächlicher Hinsicht in einer dem Rechtsgedanken des $₫ 723$ Abs. 3 BGB widersprechenden Weise eingeschränkt und der Gesellschafter aufgrund dessen zur Fortsetzung seiner Beteiligung geradezu gezwungen wird ${ }^{85}$ Hat sich eine erhebliche Diskrepanz zwischen tatsächlichem und nach dem aus der Buchwertklausel folgenden Wert aber erst nach Abschluss des Gesellschaftsvertrages herausgebildet, so kann die Inhaltskontrolle gemäß \138 Abs. 1 BGB nicht weiterhelfen, da für die Beurteilung des Rechtsgeschäfts als sittenwidrig stets der Zeitpunkt des Abschlusses des Geschäfts maßgeblich ist. ${ }^{86}$ Dennoch sind sich Rechtsprechung und Literatur weithin einig, dass auch in diesen Fällen ein gewisser Korrekturbedarf besteht. Nicht einheitlich wird dabei jedoch die Frage beantwortet, wie diese Korrektur zu erfolgen hat.

Der BGH geht - anders als früher ${ }^{87}$ - davon aus, dass eine erst nachträglich unverhältnismäßig gewordene Klausel lediglich im Wege der ergänzenden Vertragsauslegung nach $\mathbb{S} 133,157 \mathrm{BGB}$ angepasst werden müsse. ${ }^{88}$ In methodischer Hinsicht sieht $K$. Schmidt darin eine geltungserhaltende Reduktion, die am Maßstab von Treu und Glauben und mit Blick auf die Verkehrserwartung ( $(157 \mathrm{BGB})$ ausgerichtet ist. ${ }^{89}$ Das vor allem aus dem AGB-Recht bekannte Verbot der geltungserhaltenden Reduktion steht einer hier erfolgenden Vertragsanpassung aber grundsätzlich nicht entgegen. ${ }^{90}$ Das Verbot dient nur dazu, einem Vertragspartner nicht risikolos die Möglichkeit zu geben, mit im Vertrag einseitig gestellten Klauseln bis zum Äußersten zu gehen und sodann eine ggf. durch die Rechtsprechung vorgenommene Korrektur auf das noch zulässige Maß abwarten zu können. ${ }^{91}$ Bei individuell ausgehandelten (Gesellschafts-)Verträgen spielen diese Überlegungen dagegen keine Rolle.92

Betrachtet man das Vorgehen des BGH näher, so ergibt sich folgendes Bild: Zunächst wird die in Rede stehende Klausel ausgelegt und dabei als Buchwert-

85

86

87

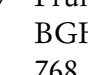

88 BGHZ 123, 281; vgl. K. Schmidt, aaO (Fn. 37), \50 IV 2 c) cc) mit Hinweis auf: DAuner-Lieb, ZHR 158 (1994), 271 ff; RASNER, ZHR 158 (1994), 292 ff; kritisch: Ulmer/ SCHÄFER, ZGR 1995, 134 ff; a. A. noch BGH NJW 1979, 104.

89 K. Schmidt, aaO (Fn. 37), \50 IV 2 c) dd).

90 BGHZ 107, 351, 355 ff; zustimmend: HENSSLER, in: Henssler/Strohn, Gesellschaftsrecht, 3. Aufl., 2016, \105 HGB Rdn. 101 m.w. N.

91 BGHZ 107, 351, 355 mit Hinweis auf BGHZ 84, 109, 116.

92 BGHZ 107, 351, 355 m.w. N. 
klausel identifiziert. Sodann wird ggf. eine erhebliche Diskrepanz zwischen dem mit der Klausel vertraglich vereinbarten Buchwertabfindungsanspruch und dem tatsächlichen Anteilswert festgestellt. Obwohl die Klausel als nach $\$ 138$ Abs. 1 BGB nichtig zu betrachten wäre, wenn die erst nachträglich eingetretenen Umstände schon bei Vertragsschluss vorgelegen hätten, ist sie nach ständiger Rechtsprechung dennoch als unwirksam zu betrachten, da eine Vereinbarung nicht abhängig vom Zeitpunkt und der Entwicklung des Unternehmens als wirksam oder unwirksam eingestuft werden dürfe. ${ }^{93}$ Der BGH macht sich damit die u.a. von K. Schmidt ${ }^{94}$ bereits früher vorgetragenen Argumente zu eigen. Zur Lösung des Problems wird die ergänzende Vertragsauslegung herangezogen. ${ }^{95}$ Die Frage sei nur, welchen Inhalt die - unverhältnismäßig gewordene - Klausel unter Berücksichtigung der Grundsätze von Treu und Glauben habe und ob sie gegebenenfalls wegen der geänderten Verhältnisse ergänzt werden müsse. ${ }^{96}$

Mit diesem Vorgehen bringt der BGH klar zum Ausdruck, dass er die Auslegung der Klausel - und sei es die ergänzende - gegenüber der Annahme der Nichtigkeit und der Ersetzung der nichtigen Klausel durch das Gesetz in jedem Falle als vorrangig erachtet. Daraus, dass er die Klausel selbst als nach \138 Abs. 1 BGB nichtig angesehen hätte, wenn die entsprechenden Umstände schon bei Vertragsschluss vorgelegen hätten, kann auch für das Verhältnis von $\mathbb{1 3 8}$ Abs. 1 BGB zur Auslegung gefolgert werden, dass sich die Auslegung und der Erhalt des Vertrages stets als vorrangig gegenüber der rechtlichen Bewertung von Nichtigkeit erweist. Für einen generellen Vorrang der Auslegung lässt sich darüber hinaus anführen, dass ein Rechtsgeschäft zumindest im Hinblick auf seinen Inhalt schon denklogisch nur dann als sittengemäß oder sittenwidrig bewertet werden kann, wenn sein konkreter Inhalt feststeht, was der vorhergehenden Auslegung bedarf.

\section{Die Inhaltskontrolle des Gesellschaftsvertrages nach $\mathbb{\int} 242 B G B$}

Als weiterer Kontrollmechanismus - zumindest eines Publikumsgesellschaftsvertrages - kommt auch eine an $₫ 242$ BGB ausgerichtete Inhaltskontrolle in Betracht. ${ }^{97}$ Vereinzelt wird sogar vertreten, dass diese unter der Anwendung

93 BGHZ 123, 281 m.w. N.

94 K. Schmidt, aaO (Fn. 37), $\ 50$ IV 2 c) cc); ebenso: RAsner, NJW 1983, 2905, 2908.

95 BGHZ 123, 281; zustimmend: Erman/Arnold, aaO (Fn. 35), \138 BGB Rdn. 35.

96 BGHZ 123, 281.

97 BGHZ 64, 238, 241; Grunewald, Münchener Komm. z. HGB, 3. Aufl., 2012, \ 161 Rdn. 124; Jaletzke, Münchener Handbuch des Gesellschaftsrechts, Bd. 2, 4. Aufl., 2014, $\$ 65$ Rdn. 8; Schubert, Münchener Komm. z. BGB, 7. Aufl., 2016, 242 Rdn. 505, 507. 
der $\int \mathbb{S} 305 \mathrm{ff}$. BGB durchgeführt werden könne. ${ }^{98}$ Methodisch zu erreichen sei dies durch eine teleologische Reduktion des $\$ 310$ Abs. 4 Satz 1 BGB. ${ }^{99}$ Für eine solche teleologische Reduktion spreche, dass die Situation bei Abschluss eines Publikumsgesellschaftsvertrages durchaus mit derjenigen vergleichbar sei, die auch beim Abschluss sonstiger Verträge bestehe, bei denen AGB verwendet werden. ${ }^{100}$

Sowohl die höchstrichterliche Rechtsprechung als auch die h.L. halten dem jedoch den zwingenden Charakter des $₫ 310$ Abs. 4 Satz 1 BGB entgegen und

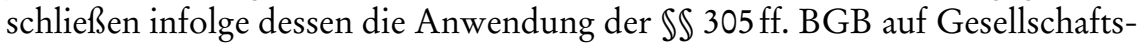
verträge insgesamt aus. ${ }^{101} \mathrm{Im}$ gleichen Atemzug befürworten sie allerdings eine über $\$ 242$ BGB begründete Inhaltskontrolle, die im Ergebnis mit der der $\int \mathbb{S} 305 \mathrm{ff}$. BGB vergleichbar sein soll und deren Wertungen daher - jedenfalls teilweise - nutzbar gemacht werden dürften. ${ }^{102}$

Inhaltlich kontrolliert die Rechtsprechung vorformulierte Gesellschaftsverträge von Publikumsgesellschaften danach, ob sie „ohne ausreichenden sachlichen Grund einseitig die Belange der Gründungsgesellschafter und Komplementäre verfolgen und unangemessen und unbillig die berechtigten Interessen der Anlagegesellschafter beeinträchtigen “. ${ }^{103}$ Die gewählte Formulierung erinnert an die des $₫ 307$ Abs. 1 BGB, der im AGB-Recht die flexibelste Form der Kontrolle darstellt. Im Rahmen der nach $\$ 242$ BGB durchzuführenden Inhaltskontrolle müssen insbesondere auch die individuellen Umstände und damit die jeweiligen Verhältnisse der Gesellschaft berücksichtigt werden. ${ }^{104}$ Diese Sichtweise kann damit begründet werden, dass maßgebliches Kriterium der Inhaltskontrolle die "Treue“ ist, die eine Seite der anderen schuldet, und es bei der „Treue“ um das gesamte Verhältnis zwischen den Vertragsparteien und damit auch um ihre individuelle Beziehung geht. ${ }^{105} \mathrm{Maßstab}$ der Inhaltskontrolle soll daher auch die gesellschaftsrechtliche Treuepflicht ${ }^{106}$ sein, über die insbesondere die jeweiligen Verhältnisse der Gesellschafter untereinander Be-

98 OLG Oldenburg NZG 1999, 896; KäHLER, in: BeckOGK-BGB, Stand 1.8.2017, $\$ 242$ Rdn. 911 m.w. N.; Servatius, in: Henssler/Strohn, Gesellschaftsrecht, 3. Aufl., 2016, Publikumsgesellschaft Rdn. 9 m.w.N.

99 Kähler, aaO (Fn. 98), $\ 242$ BGB Rdn. 911 m.w. N.; Lehmann-Richter, in: Graf v. Westphalen, Vertragsrecht, 38. El. 2016, Publikumsgesellschaften Rdn. 8.

100 KÄhleR, aaO (Fn. 98), \$242 BGB Rdn. 911 m. w. N.

101 BGHZ 128, 93; BGHZ 64, 238, 241; MAYER, aaO (Fn. 48), $\ 2$ GmbHG Rdn. 18.

102 BGHZ 128, 93; BGHZ 64, 238, 241; JaletZke, aaO (Fn. 97), \$65 Rdn. 10.

103 OLG Düsseldorf BeckRS 2007, 12068; BAsedow, Münchener Komm. z. BGB, 7. Aufl., 2016, $\$ 310$ Rdn. 87 m. w. N.; JaletZke, aaO (Fn. 97), 65 Rdn. 8 m. w. N.

104 KÄHLER, aaO (Fn. 98), \242 BGB Rdn. 896.

105 KÄHLER, aaO (Fn. 98), \242 BGB Rdn. 126.

106 Schubert, aaO (Fn. 97), \242 BGB Rdn. 507. 
rücksichtigung finden. ${ }^{107}$ Im Interesse der Vertragsparteien geht rechtsfolgenseitig jedoch die geltungserhaltende Reduktion der Nichtigkeitsfolge vor. ${ }^{108}$

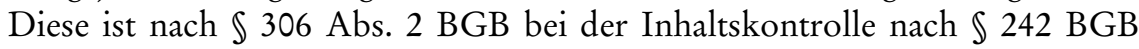
anders als bei einer solchen nach $\mathbb{\int} 305 \mathrm{ff}$. BGB gerade nicht ausgeschlossen ${ }^{109}$ und bietet demgemäß den Vorteil, eine an sich unwirksame Klausel dem durch Auslegung ermittelten Parteiwillen entsprechend auf das noch Zulässige anzupassen.

Bedeutung für die vorliegende Untersuchung erlangt die an $\$ 242$ BGB ausgerichtete Inhaltskontrolle unter Berücksichtigung zweier Aspekte. Zum einen erscheint es aufgrund der hinter ihr stehenden Schutzteleologie möglich, diese auch auf den Gesellschaftsvertrag einer solchen Familiengesellschaft anzuwenden, die angesichts der fehlenden Anonymität der Gesellschafter untereinander zwar nicht als Publikumspersonengesellschaft einzuordnen sein dürfte, die jedoch - beispielsweise aufgrund von mehreren Erbfällen - zu einer Gesellschaft mit einer Vielzahl von Gesellschaftern geworden ist. ${ }^{110}$ Zum anderen aber vor allem auch dadurch, dass im Rahmen einer solchen Inhaltskontrolle die Möglichkeit besteht, auch individuelle Verhältnisse und damit gegebenenfalls auch eine eventuell existente Familienverfassung in der dabei durchzuführenden Angemessenheitskontrolle zu berücksichtigen und den Gesellschaftsvertrag damit auch anhand einer solchen zu überprüfen.

Ebenfalls von Interesse ist der Umstand, dass die Wertungen der $\$ \mathbb{S} 305 \mathrm{~b}$ und 305 c BGB im Rahmen der Inhaltskontrolle nach $\$ 242$ BGB zu berücksichtigen sein sollen. ${ }^{111}$ Zur dogmatischen Begründung dieser Sichtweise lässt sich anführen, dass die $\$ \mathbb{S} 305 \mathrm{ff}$. BGB lediglich eine gesetzgeberische Konkretisierung der in $\$ 242$ BGB normierten Grundsätze von Treu und Glauben darstellen $^{112}$ und sich deren Wertungen daher $\ 242$ BGB schon selbst entnehmen lassen müssten. Geht man danach davon aus, dass auch die Wertungen der $\mathbb{\int} \int 305 \mathrm{~b}, 305 \mathrm{c}$ BGB Teil der an $\$ 242$ BGB ausgerichteten Inhaltskontrolle sind, so ist zu erwägen, ob die Familienverfassung eine dem Gesellschaftsvertrag nach der Wertung des $\$ 305$ b BGB vorgehende „Individualvereinbarung“

107 Roth, in: Baumbach/Hopt, Komm. z. HGB, 37. Aufl., 2016, $\$ 109$ Rdn. 23; Drescher, in: Henssler/Strohn, Gesellschaftsrecht, 3. Aufl., 2016, $\$ 243$ AktG Rdn. 22.

108 BGH NJW 2006, 2854; Lehmann-Richter, aaO (Fn. 99), Publikumsgesellschaften Rdn. 19.

109 Lehmann-Richter, aaO (Fn. 99), Publikumsgesellschaften Rdn. 14; Henssler, aaO (Fn. 90), $₫ 105$ HGB Rdn. 101.

110 Grunewald, aaO (Fn. 97), $\ 161$ HGB Rdn. 107; a. A.: Häublein, in: BeckOK HGB, Stand 1.11.2016, $\mathbb{1} 161 \mathrm{Rdn} .75$.

111 BGH BeckRS 2013, 22860; BAsedow, aaO (Fn. 103), $\$ 310$ BGB Rdn. $87 f$ m.w.N.; Schubert, aaO (Fn. 97), \242 Rdn. 504, 507; Servatius, aaO (Fn. 98), Publikumsgesellschaft Rdn. 12; Jaletzke, aaO (Fn. 97), \$ 65 Rdn. 6.

112 BGHZ 128, 93 = NJW 1995, 583. 
darstellen könnte, wenn sich Gesellschaftsvertrag und Familienverfassung widersprechen und sämtliche Gesellschafter zumindest auch von der Familienverfassung Kenntnis genommen und ihrer Berücksichtigung auf diese Weise wenigstens stillschweigend zugestimmt haben. Individualabreden setzen weder Schriftform noch eine ausdrückliche Vereinbarung voraus. ${ }^{113}$ Zudem ist es für ihren Vorrang unerheblich, ob sie zeitlich vor oder erst nach Vertragsschluss getroffen wurden. ${ }^{114}$ Einzig problematisch erscheint, dass die Individualvereinbarung i.S.d. $\$ 305$ b BGB eine „Vertragsabrede“ ${ }^{115}$ und damit ein „wirksames Rechtsgeschäft" ${ }^{\text {"116 }}$ voraussetzt und den bei Abschluss der Familienverfassung Beteiligten - zumindest nach verbreiteter Ansicht - ein Rechtsbindungswille abgesprochen und damit auch die Einordnung der Familienverfassung als Rechtsgeschäft überwiegend abgelehnt wird. ${ }^{117}$ Dem zum Trotze hat Fleischer jüngst aufgezeigt, dass die Auslegung einer Familienverfassung zu der Annahme eines Rechtsbindungswillens der daran Beteiligten führen kann, ${ }^{118}$ was insbesondere in jenen Fällen unmittelbar einleuchtet, in denen die Beteiligten der Urkundenerrichtung Regelungen für allseits erkannte oder eindeutig erkennbare relevante Regelungsgegenstände aufnehmen. So kann etwa bezüglich einer Nachfolgeregelung mit Qualifikationsanforderungen für die Geschäftsführung des Unternehmens kaum noch durch die Parteien der Charta argumentiert werden, dass man mit der Niederlegung keine für die übrigen Teilnehmer verlässliche und allseits bindende Vereinbarung habe schaffen wollen.

Abstrakt gesprochen, besteht jedenfalls dahingehend Einigkeit, dass die Auslegung auch einer an $\$ 242 \mathrm{BGB}$ ausgerichteten Inhaltskontrolle vorgeht, da eine solche nur als ultima ratio in Betracht kommt. ${ }^{119}$ Dementsprechend ist im Wege der Auslegung zunächst dem Willen aller Beteiligten Rechnung zu tragen und eine Inhaltskontrolle erst im Anschluss vorzunehmen, wenn die Auslegung - und sei es auch eine ergänzende -, an ihre Grenzen gestoßen ist. ${ }^{120}$ Das folgt schon daraus, dass eine korrekte Inhaltskontrolle nur dann vorgenommen werden kann, wenn der konkrete Inhalt durch Auslegung er-

113 Schulte-Nölke, in: HK-BGB, 9. Aufl., 2017, $\$ 305$ b Rdn. 3; Basedow, aaO (Fn. 103), $\$ 305$ b BGB Rdn. 5 .

114 BGH NJW 2006, 138; Schulte-Nölke, aaO (Fn. 113), \305 b BGB Rdn. 3; Basedow, aaO (Fn. 103), $₫ 305$ b BGB Rdn. 5.

115 Basedow, aaO (Fn. 103), $\$ 305$ b BGB Rdn. 5.

116 Schulte-Nölke, aaO (Fn. 113), $\$ 305$ b BGB Rdn. 3.

117 FleisCher, ZIP 2016, 1509, 1511 m. w. N.

118 FLeISCHER, ZIP 2016, 1509, 1516 m.w. N.

119 BGH NJW 1979, 2102, 2103; HenZe/NotZ, in: Ebenroth/Boujong/Joost/Strohn, HGB, 3. Aufl., 2014, Anhang B Rdn. 31; Lehmann-Richter, aaO (Fn. 99), Publikumsgesellschaften Rdn. 13; Servatius, aaO (Fn. 98), Publikumsgesellschaft Rdn. 12.

120 K. Schmidt, aaO (Fn. 62), $\$ 131$ HGB Rdn. 157. 
mittelt worden ist. ${ }^{121}$ Folgendes Rechtsprechungsexempel soll diesen zentralen Gedanken de lege lata noch erhärten: Eine gegen $\ 9$ AGBG verstoßende, weil ermessensabhängige und daher unwirksame Freigabeklausel einer Sicherungsübertragung soll nicht die Gesamtnichtigkeit der Sicherungsübertragung zur Folge haben. Vielmehr soll an die Stelle der unwirksamen Klausel im Wege der ergänzenden Vertragsauslegung eine ermessensunabhängige Klausel treten, mit deren Hilfe die Sicherungsübertragung aufrecht erhalten werden könne. Dieser ergänzenden Vertragsauslegung stehe auch das Verbot der geltungserhaltenden Reduktion nicht entgegen, da die ergänzende Vertragsauslegung ihrem Rechtscharakter nach dispositivem Gesetzesrecht gleichstehe.

Der BGH verdeutlicht, dass die (ergänzende) Vertragsauslegung auch der Anordnung der aus AGB-Kontrolle folgenden Nichtigkeit des Vertrages vorgeht. Mit Hilfe eines Erst-Recht-Schlusses dürfte dies gleichermaßen auf eine an $\ 242$ BGB ausgerichtete, AGB-Kontrollen-ähnliche Inhaltskontrolle zu übertragen sein. Die erkennbaren Parallelen der skizzierten BGH-Entscheidungen zu den sog. Buchwertklauseln auf der einen und zu den ermessensabhängigen Freigabeklauseln auf der anderen Seite lassen den Schluss zu, dass sich die Auslegung stets gegenüber den anderweitig denkbaren und anerkannten bürgerlich- und gesellschaftsrechtlichen Kontrollmechanismen als vorrangig erweist und der Parteiwille Vorrang genießt.

\section{Trenepflicht}

Schließlich kommt als gesellschaftsrechtsspezifischer „Kontrollmechanismus“ auch die Treuepflicht der Gesellschafter in Betracht. Besonders im neueren Schrifttum ist zu lesen, dass auch eine Familienverfassung geeignet sei, die generalklauselartig ${ }^{122}$ ausgestaltete Treuepflicht näher zu konkretisieren und der Familienverfassung auf diese Weise besondere Geltung im Gesellschaftsrecht zu verschaffen. ${ }^{123}$ Dieser (Um-)Weg muss jedoch nur dann beschritten werden, wenn entweder feststünde, dass etwa eine Familienverfassung über die Auslegung des Gesellschaftsvertrages nicht berücksichtigt werden kann, was allerdings grundsätzlich nicht der Fall sein dürfte, oder wenn die Treuepflicht der Auslegung vorginge.

Die gesellschaftsrechtliche Treuepflicht verpflichtet die Gesellschafter, sich gegenüber der Gesellschaft und den anderen Mitgesellschaftern loyal zu verhalten, die Zwecke der Gesellschaft zu fördern, Schaden von ihr abzuhalten und auf die mitgliedschaftlichen Interessen der Mitgesellschafter Rücksicht

123 Fleischer, ZIP 2016, 1509, 1519 m.w.N. 
zu nehmen. ${ }^{124}$ Wird gegen sie verstoßen, sind - je nach Bedarf - verschiedene Rechtsfolgen denkbar: Neben der Möglichkeit der Beschlussanfechtung von $\mathrm{GmbH}-G e s e l l s c h a f t e r b e s c h l u ̈ s s e n^{125}$ und über $\ 280$ Abs. 1 BGB begründeten Schadensersatzansprüchen ${ }^{126}$ kommt als ultima ratio auch der Ausschluss eines Gesellschafters ${ }^{127}$ in Betracht. ${ }^{128}$ Dieses Zusammenspiel von Tatbestand und Rechtsfolgen zeigt, dass es sich bei der Treuepflicht um einen nachträglich korrigierenden Kontrollmechanismus handelt. In diesem Fall dürfte sich der zuvor bereits festgestellte allgemeingültige Grundsatz, wonach die Auslegung der Inhaltskontrolle bereits bestimmter Erklärungen vorgeht, auch auf das Verhältnis von Auslegung und Treuepflicht übertragen lassen. Allerdings greift die Treuepflicht nach h.M. in praxi nicht nur nachträglich ein. Beispielsweise kann mit ihrer Hilfe auch eine Handlungspflicht der Gesellschafter begründet werden. ${ }^{129}$ Wird dieser nicht nachgekommen, so kann nach h.M. auf Erfüllung geklagt werden. ${ }^{130}$ Es handelt sich dabei folglich um eine allgemeine, an sämtliche Gesellschafter gerichtete Verhaltensregel. ${ }^{131}$ Die Treuepflicht ist eine Generalklausel des Richterrechts, mit deren Hilfe der Einzigartigkeit jeder Gesellschaft und der damit zwangsläufig verbundenen notwendigen Lückenhaftigkeit des Gesellschaftsrechts begegnet werden kann. ${ }^{132}$

Das Verhältnis zur Auslegung kann über die dogmatische Einordung der Treueplicht ${ }^{133}$ erfasst werden:

Mitunter wird die Treuepflicht der Gesellschafter als „wichtige Auslegungsmaxime" bezeichnet. ${ }^{134}$

124 Wicke, GmbHG, 3. Aufl., 2016, $\mathbb{1 3}$ Rdn. 19; Fastrich, aaO (Fn. 69), $\ 13$ GmbHG Rdn. 21.

125 BGHZ 76, 357; Fastrich, aaO (Fn. 69), $\$ 13$ GmbHG Rdn. 30.

126 BGHZ 65, 15, 21.

127 BGHZ 9, 157, 163.

128 Wicke, aaO (Fn. 124), $\mathbb{1} 13$ GmbHG Rdn. 21; Fastrich, aaO (Fn. 69), $\$ 13$ GmbHG Rdn. 30.

129 Grunewald, aaO (Fn. 66), $\ 1$ Rdn. 19; SchäFer, aaO (Fn. 68), $\$ 9$ Rdn. 9.

130 Staub/SchäFer, Großkomm. z. HGB, 5. Aufl., 2008, \105 Rdn. 228, 230; Lutter, AcP 180 (1980), 84, 118; Grunewald, aaO (Fn. 66), $\$ 1$ Rdn. 23 m.w. N.

131 Pentz, in: Rowedder/Schmidt-Leithoff, Komm. z. GmbHG, 6. Aufl., 2017, $\ 13$ Rdn. 38; zustimmend: FASTRICH, aaO (Fn. 69), $\$ 13$ GmbHG Rdn. 20.

132 HÜFFER, FS Steindorff, 1990, S. 59, 73.

133 Wesen und Hintergrund der Treuepflicht sind nach wie vor streitig, vgl. MerkT, Münchener Komm. z. GmbHG, 2. Aufl., 2015, $\mathbb{1} 13$ Rdn. 95 f; Fastrich, aaO (Fn. 69), \13 GmbHG Rdn. 20.

134 Heidel, in: NK-BGB, 3. Aufl., 2016, $\$ 705$ Rdn. 174; vgl. auch: Heidel, in: Heidel/ Schall, HGB, 2. Aufl., 2015, $\mathbb{1} 105$ Rdn. 229 m.w. N. 
Hält man dem jedoch entgegen, dass die Treuepflicht nach überwiegender Ansicht ungeschriebener Inhalt ${ }^{135}$ des Gesellschaftsvertrages ist, so ist die Einordnung der Treuepflicht als Auslegungsmaxime schwer haltbar. Vielmehr muss der Gehalt der Treuepflicht selbst erst durch Auslegung extrapoliert werden. Sieht man also die Grundlage der Treuepflicht nach der auch hier vertretenen Auffassung in der durch die Mitgliedschaft begründeten engen Verbundenheit der Gesellschafter und damit im Gesellschaftsvertrag, so ist sie, da sie selbst der Konkretisierung durch Auslegung bedarf, nichts anderes als das Produkt der Auslegung. Dieses Verständnis dürfte auch die einzig verfassungskonforme Verständnisvariante mit Blick auf das Wesen der Privatautonomie und der Vereinigungsfreiheit darstellen. Mangels gesetzlicher Anordnung der allgemeinen Treuepflicht kann sie sich nur auf den eigenverantwortlichen und zurechenbaren Bindungsbeschluss aller Gesellschafter bei Vertragserrichtung stützen, der mithin der zentrale Ansatz für das Verständnis von Inhalt und Reichweite zu sein hat. Man mag Modifikationen, insbesondere im Fall von Publikumsgesellschaften erwägen, jedoch kann auch für diesen Fall der Treuepflichtgedanke für jeden Einzelfall auf Gründung von oder Beitritt zu einer solchen Gesellschaft respektive Verbleiben in derselben bei Vergrößerung des Gesellschafterkreises zurückgeführt werden. Da die Treuepflicht zudem als rechtsformübergreifendes allgemeines Konstrukt des Gesellschaftsrechts verstanden wird, ${ }^{136}$ kann deren dogmatische Basis auch gerade nicht von Eigenheiten eines spezifischen Typus abhängig sein, sondern allenfalls den durch Auslegung des Gesellschaftsvertrages und seiner Umgebungsvariablen erkannten Inhalten folgen oder diese notfalls modifizieren. ${ }^{137}$

\section{Zwischenergebnis}

Damit ist festzuhalten, dass die Auslegung sowohl den allgemeinen bürgerlich-rechtlichen Kontrollmechanismen als auch der gesellschaftsrechtlichen Treuepflicht vorgelagert ist. Die Beantwortung der Frage nach Wechselwirkungen zwischen Gesellschaftsvertrag und anderen normativen Ebenen - etwa einer Familienverfassung - muss somit die treffende Auslegung des Gesellschaftsvertrages zum Ausgangspunkt haben.

135 BGH NJW 2011, 1667, 1669; WerTenBruch, Ebenroth/Boujong/Joost/Strohn, HGB, 3. Aufl., 2014, \105 Rdn. 103; Ulmer/SchäFer, Münchener Komm. z. BGB, 6. Aufl., 2013, $\ 705$ Rdn. 222; HüFfer, FS Steindorff, S. 59, 65 m.w.N.; Grunewald, aaO (Fn. 66), $\$ 1$ Rdn. 13, 17.

136 Born, in: Ebenroth/Boujong/Joost/Strohn, HGB, 3. Aufl., 2014, $\mathbb{1 0 9}$ Rdn. 20 m. w. N.; HÜFFER, FS Steindorff, S. 59, 65.

137 Vgl. Heidel, aaO (Fn. 142), \ 705 BGB Rdn. 195 mit Hinweis auf Flume, Personenge-

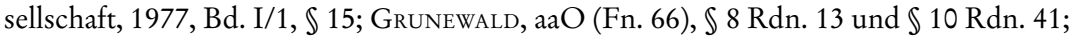
StAub/SCHÄFer, aaO (Fn. 130), $\$ 105$ HGB Rdn. 242. 
V. Spezifika des Gesellschaftsrechts vs. begleitende Vereinbarungenzwingender Schutz durch Gesellschaftsrecht ${ }^{138}$

Auf die obigen Erwägungen aufsetzend ist schließlich die zentrale Frage zu beantworten, welcher Einfluss begleitenden Vereinbarungen und außerstatutarischen Umständen im Rahmen der Auslegung letztlich zukommen kann. Schon aufgrund der sachlichen Nähe ist auch diese Rechtsfrage nur vom Anschauungsobjekt Gesellschaftsvertrag her zu beantworten, um Elemente eines zwingenden und damit statischen Schutzes von dynamischen und mithin beeinflussungsfähigen Gesellschaftsrechtsgegenständen abzugrenzen.

Die Diskussion um zwingende Schutzelemente ist im Bereich des Gesellschaftsrechts mit den Regelungen zu Gründung, Stellvertretung und Haftung sowie Beendigungstatbeständen bestens bekannt. Hintergrund des ius cogens ist insoweit der unübersehbare Verkehrsschutz zu Gunsten Dritter, die mit der Gesellschaft als Rechtsperson interagieren und weder rechtsgeschäftlichen Einfluss auf Vertretungs- und Haftungsstruktur derselben zu nehmen vermochten noch im Fall der Anerkennung dispositiver Regelungen mit Außenwirkung hinreichende Transparenz und Information zu ihrem Schutz für sich verbuchen könnten. ${ }^{139}$ Es liegt entsprechend für die hier geführte Debatte nahe, nach ähnlich scharfen Begrenzungsargumenten im Innverhältnis der Gesellschaft zu suchen. Dabei sind insbesondere die gesetzgeberisch vorgegebenen konzeptionellen Unterschiede zwischen Personen- und Kapitalgesellschaften kritisch und ergebnisoffen zu berücksichtigen.

\section{Akute Drittbetroffenheit (einheitliche vs. gespaltene Auslegung)}

Soweit Gesellschafter Anteile halten, die dem zurechenbaren Kreis gesellschaftsvertragsexterner Umstände nicht angehören, erscheinen diese Personen zunächst ebenso schutzwürdig und schutzbedürftig wie der sonstige Rechtsverkehr, der mit der Gesellschaft interagiert. So wird sich nach den anerkannten Auslegungskriterien auch mit Blick auf eine familiengetragene Gesellschaft nicht argumentieren lassen, dass ein Investor, der gesellschaftsvertraglich zugesicherten Einfluss auf Gewinnverwendungsregelungen inne hat, diese Vorschiften im Lichte modifizierender respektive zumeist begrenzender Bestimmungen der Familienverfassung zu befolgen hätte, solange keine Ein-

138 Unter dem Stichpunkt „reiner Beteiligtenbetroffenheit“ sollte neben den unten erörterten Aspekten der akuten und der potentiellen Drittbetroffenheit noch eine ausführliche Erörterung der Vererblichkeit von Wissen eingebunden werden. Diese muss aus Gründen des Umfangs jedoch einer eigenständigen Ausarbeitung vorbehalten bleiben.

139 Besonders deutlich in der gesetzgeberischen Wertung des $\$ 128$ Satz 2 HGB hervorgetreten, vgl. hierzu K. Schmidt, aaO (Fn. 62), \128 HGB Rdn. 13. 
beziehung in das außerstatutarische Vertragswerk vorliegt oder jedenfalls ein Zurechnungstatbestand angeführt werden könnte. Damit ist jedoch Dreh- und Angelpunkt, was genau als Zurechnungstatbestand genügen kann.

\section{a) Zurechnungstatbestände bei Dritten}

Eine kaum bestreitbare Einbeziehung stellt zunächst die Beteiligung des Dritten an der außerstatutarischen Vereinbarung, etwa in Form der formellen Aufnahme in dieses Vertragswerk oder durch einen Neuabschluss dar. Besonderheiten ergeben sich insoweit allerdings dort, wo selbst die außerstatutarische Regelung einen interpretationsoffenen Inhalt aufweist, den die Familienmitglieder aufgrund der Vertragsgenese oder innerfamiliärer Gepflogenheiten anders verstehen mögen, als dies nach außen erkennbar wäre. Die üblichen Grundsätze der Auslegung führen diesbezüglich ohne weiteres zu sachgerechten Ergebnissen, da nunmehr der gesetzliche Regelfall des objektiv schutzwürdigen Empfängerhorizonts gemäß $\$ 157$ BGB eingreift, der sich nur daran festhalten lassen muss, was für den durchschnittlichen Empfänger in eben jener Situation verständlich gewesen wäre. ${ }^{140}$

Komplexer stellt sich demgegenüber die Fragestellung dar, ob ein Dritter auch durch schlichtes Wahrnehmen oder gar durch die bloße Möglichkeit zur Kenntnisnahme der Familienverfassung oder anderer Nebenvereinbarungen an ihren erkennbar niedergelegten Inhalt gebunden sein kann und eine Modifikation des Gesellschaftsvertragsverständnisses hinzunehmen haben könnte. An dieser Stelle muss die Rechtsordnung Farbe bekennen, welchen Vertrauensschutz Mitgesellschafter untereinander mit Blick auf Begleitumstände und Vertragsgenese haben sollen und wie viel Sorgfalt einem hinzutretenden Dritten zuzumuten ist. Letztlich besteht Einigkeit darüber, dass die Entscheidung einer umfassenden Abwägung des Einzelfalls vorbehalten sein dürfte. ${ }^{141}$ Prozessual formuliert: Die Macht der Letztentscheidung liegt regelmäßig beim Tatrichter, obgleich gerade dieser mit Blick auf Art und Inhalte der juristischen Ausbildung ganz überwiegend einem Hang zur objektiven Auslegung nachgeben wird, da diese die Entscheidung erheblich vereinfacht und dem Tatrichter die Möglichkeit gewährt, den aus seiner Sicht möglicherweise absurden Gesellschafterstreit mit Mitteln der eigenen Logik und eigener Wertvorstellungen zu begradigen, sich also letztlich nicht auf den schwierigen und dichten Pfad der Ergründung des wahren Gesellschafterwillens einlassen zu müssen.

140 Detailliert zur vorzugswürdigen Geltungstheorie Thomale, Leistung als Freiheit, 2012, S. $59 \mathrm{ff}$ m.w. N. Ein eigenständiger dogmatischer Streit um die Abgrenzung von Empfänger- und Geltungstheorie ist für die vorliegende Debatte nicht geboten.

141 Vgl. BGHZ 9, 157, 163; 14, 25, 38; 51, 204, 206; 65, 15, 19, 98, 276, 279f; BGH ZIP 2010, 1541; MAyer, aaO (Fn. 48), 22 GmbHG Rdn. $150 \mathrm{ff}$ m.w. N. 
Die damit einhergehende Diskrepanz zwischen gesellschaftsvertraglich vorgezeichnetem und letztlich im Streitfall erhaltenem Ergebnis sowie die damit für die Parteien kaum zu antizipierende fortbestehende Dynamik einer später zur Entscheidung berufenen dritten Instanz ist wohl nicht vollends aus der Welt zu schaffen, jedoch können ihr richtungsweisende Parameter an die Seite gestellt werden, die ihrerseits Rechtsfragen und mithin der Revision zugänglich sind. Die Konsequenz des Gelingens dieses Unterfangens wäre, dass der Tatrichter sich bei Vorliegen dieser Parameter gezwungen sähe, den Ideen der gewachsenen gesellschaftsrechtlichen Struktur nachzuspüren. In der Sache weist Holler mit Recht darauf hin, dass einer lediglich erkennbaren „Grundtendenz ${ }^{\text {" } 142}$ des Gesellschaftsvertrages auch nach Ansicht der höchstrichterlichen Rechtsprechung de lege lata im Rahmen der Auslegung Aufmerksamkeit $\mathrm{zu}$ schenken sein dürfte. ${ }^{143}$ Ein familiengetragenes Unternehmen weist eine solche Tendenz zu begleitenden Vereinbarungen, insbesondere zu Regelungen im Bereich Nachfolgeplanung, Gewinnverwendung und Zukunftsausrichtung, Streitschlichtung zwischen Familienstämmen, Anforderungen an die Besetzung der Geschäftsführung, Wertebindung und zahlreiche vergleichbar an die Familieninteressen gebundene Aspekte, regelmäßig auf. ${ }^{144}$

Diese Elemente und ihre im Einzelfall gewählte Regelungsstruktur durch die Gründungsgesellschafter werden generationenübergreifend weitergegeben und erhalten. Eben hierauf weist auch Holler in seiner Analyse trefflich hin, wenn er sich bei der Familiengesellschaft gegen ein zu berücksichtigendes unabhängiges Eigenleben ${ }^{145}$ von Familiengesellschaften wendet und auf die Besonderheiten der gewählten Realstruktur verweist. ${ }^{146}$ Jedoch kann das rein tatsächliche Vorliegen einer solchen Tendenz-Gesellschaft für sich nicht genügen. Als entscheidende Transparenz- und mithin letztlich argumentative $\mathrm{Zu}$ rechnungskriterien für eine anzuerkennende Nachforschungsobliegenheit des hinzutretenden Dritten sind die gesellschaftsvertragliche Präambel und der erklärte Gesellschaftszweck, Nennung von außerstatutarischen Klauselwerken im Gesellschaftsvertrag, für jedermann nach außen erkennbare bisherige Inhaberschaft einer Unternehmerfamilie, strikte Vererbungsregelungen für Familiengesellschafter und die Gesellschaftsgeschichte sowie ihre Gesellschafter zu nennen. $\mathrm{Zu}$ diesen letzten beiden Aspekten sei näher ausgeführt, dass ein Dritter, der Gesellschafter wird, in seinem Unwissen über Gründung und Entwicklung der Gesellschaft nicht einem sonstigen Dritten im Rechtsverkehr gleichgestellt werden darf. Mit der Gesellschafterstellung gehen die geschrie-

142 So BGH NJW 1987, 890.

143 Holler, aaO (Fn. 9), $\$ 75$ Rdn. 132 f.

144 Hierzu bereits oben unter II. 2.

145 So die Argumentation der Rspr. in BGH NJW-RR 1989, 1259f; 1989, $993 \mathrm{f}$.

146 Holler, aaO (Fn. 9), $\mathbb{\int} 75$ Rdn. 132 im Rahmen der Diskussion über subjektive vs. objektive Auslegung bei großen Familiengesellschaften. 
benen wie ungeschriebenen gesellschaftsrechtlichen Rechte und Pflichten einher, insbesondere Auskunfts- und Informationsmöglichkeiten, gleichermaßen Anspruch auf Bilanzgewinn respektive wirtschaftliche Vorteile und die Pflicht zur Abwendung von Schaden. Auf den Punkt gebracht: Gesellschafter suchen einander als besonders enge Rechtsgemeinschaft aus, nehmen die einhergehenden Vorteile in Anspruch und müssen konsequenterweise die Lasten dessen tragen. Wer vor diesem Hintergrund im Rahmen der Vertragsanbahnung erkennen kann, dass er Teil einer in sich geschlossenen, klar umgrenzten und nach einer familiären Struktur ausgerichteten Organisation werden soll, kann nach gesetzlichem Maßstab des $\$ 157$ BGB und damit von jedermann geforderter Beachtung allgemeiner Lebenserfahrung nicht für sich in Anspruch nehmen, keinen außerstatutarischen Einfluss auf Unternehmensangelegenheiten erwartet zu haben.

Daraus lässt sich sicherlich keine Rechtspflicht im engeren Sinne deduzieren, da es einem hinzutretenden Gesellschafter de lege lata ohne Weiteres erlaubt ist, sich „blind“ auf neue Gegebenheiten einzulassen und auch rechtliche Verpflichtungen mitzutragen, die ihm im Einzelnen nicht bekannt sind. Allerdings handelt es sich um eine Obliegenheit, deren fehlende Wahrnehmung zur gerade genannten pauschalen Akzeptanz führen muss. Für dieses Ergebnis sprechen auch parallele gesetzliche und in der Rechtsprechung anerkannte Wertungen. So basiert die aktuelle höchstrichterliche Rechtsprechung zur Wissenszurechnung innerhalb von Organisationen wesentlich auf der Möglichkeit, sich aus wahrnehmbarem Aktenstudium kundig zu machen. ${ }^{147}$ Hinzu tritt eine Obliegenheit zur Schaffung einer Struktur, die vernunftgeleitete Weitergabe von relevatem Wissen ermöglicht und befördert. ${ }^{148}$ Diese Elemente verlangen die Nutzung abstrakt zu erwartender und regelmäßig konkret bestehender Informationsquellen. Ein weiterer Leitgedanke lässt sich $\mathbb{} 142$ Abs. 2 BGB entnehmen. Wissen, welches erhebliche rechtliche Negativkonsequenzen wie die Kenntnis der Nichtigkeit eines Rechtsgeschäfts zeitigt, wird dem Wissen der nichtigkeitsbegründenden Umstände gleichgestellt. Entsprechendes gilt auch für die Rechtsprechung zur relevanten Kenntnis sittenwidrigkeitsbegründender Umstände in $\ 138$ BGB.$^{149}$ Gesetz und Rechtsprechung verlangen mithin selbst mit Blick auf die Konsequenz der Nichtigkeit grundsätzlich nur die Kenntnis der sie begründenden Umstände, was in einem Rückschluss a maiore ad minus für gesellschaftsvertraglich zurechenbares Verständnis die Kenntnis der auslegungsrelevanten Gegebenheiten als hinreichend erscheinen lässt.

147 BGHZ 135, 202, 205; BGHZ 109, 327, 331; Freitag, in: Heidel/Schall, HGB, 2. Aufl.,

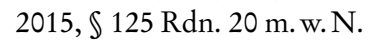

148 Wisskirchen/Kuhn, in: BeckOK GmbHG, 30. Ed. 2017, \35 Rdn. 105.

149 BGHZ 146, 301; BGH WM 1998, 513, 514; MANSEL, aaO (Fn. 77), \138 BGB Rdn. 11. 
Eine besonders faszinierende Nähe zur vorliegenden Problematik weisen schließlich $\int \mathbb{S} 133$ Abs. 2 und 138 InsO (bzw. $\$ 3$ Abs. 4 AnfG) auf, die nahestehende Personen per Gesetz im Fall der Insolvenzanfechtung ohne andernfalls nach $₫ 133$ Abs. 1 InsO erforderlichen Wissensnachweis diskriminieren. Besonderes Augenmerk gilt dabei $\mathbb{} \$ 138$ Abs. 1 Nr. 4 InsO, welcher eine Gesellschaft legaldefinitorisch unter die nahestehende Person fasst, sofern der Schuldner persönlich haftender Gesellschafter oder Teil des Leitungs- oder Aufsichtsorgans ist. Selbstverständlich wird an dieser Stelle nicht die gläubigerschützende Teleologie der Insolvenzordnung verkannt. Es ergibt sich aus besagter Einordnung vielmehr ein interessanter Gegenschluss auf die Nähe und die Obliegenheiten der Gesellschafter, die ihre Gefahren in die Gesellschaft und damit zwangsläufig in den Gesellschafterkreis tragen, dementsprechend aber gleichermaßen ihrer Gesellschaft wie auch ihren Mitgesellschaftern gegenüber die Obliegenheit akzeptieren müssen, sich den kognitiven Besitzstand anzueignen, der zum erkennbaren und zutreffenden Verständnis des status quo ante der Gesellschaft führt. Der Gesellschaftsvertrag ist dabei der erste und zentrale Anlaufpunkt.

Dieses vermeintlich scharfe und einseitig Neugesellschafter belastende Zurechnungsmodell erfährt jedoch prozessual eine weitreichende Abmilderung über Darlegungs- und Beweislastgrundsätze, da sich in einem entsprechenden Gesellschafterstreit die im Gesellschaftsvertrag niedergelegten Regelungen durch Urkundenvorlage ohne Weiteres darlegen und beweisen lassen, während im Übrigen jeder die Darlegungs- und Beweislast dafür trägt, dass eine behauptete außerstatutarische Modifikation respektive Auslegungsvariante zu berücksichtigen sei und dass die hiervon negativ betroffenen, später hinzugetretenen Neugesellschafter sich anhand der oben genannten Zurechnungskriterien nicht sorgfältig genug informiert hätten. ${ }^{150}$

\section{b) Sonderauslegung für und gegen Beteiligte der außerstatutarischen Vereinbarung - einheitliche vs. gespaltene Auslegung}

Das bisherige Ergebnis mit Blick auf Drittbetroffene enthält keine zwingende Aussage für das Verständnis des Gesellschaftsvertrages zwischen den Beteiligten der Familienverfassung respektive der sonstigen außerstatutarischen Vereinbarung. Es stellt sich konsequenterweise die Frage, ob der Gesellschaftsvertrag in den Fällen akuter Drittbetroffenheit und im Einzelfall mangels nachweislichen Zurechnungskriterien anzuerkennendem Schutz der Unwissenheit des Dritten im Übrigen zu einer einheitlichen oder einer gespaltenen Auslegung führt. Weder im Recht der Gesamthandsgemeinschaften noch im 
Recht der Korporationen findet sich ein klarer Hinweis auf eine gesetzgeberische Vorstellung für oder gegen eine gespaltene Auslegung.

Keinen erschöpfenden Ansatz bietet dabei die Unterscheidung zwischen Personen- und Kapitalgesellschaften, wie nicht zuletzt der BGH in der Kerbnägel-Entscheidung ${ }^{151}$ selbst zu Protokoll gegeben hat. Die Grundtendenz der Rechtsprechung, im Kapitalgesellschaftsbereich ${ }^{152}$ mit Blick auf Formerfordernisse des Gesellschaftsvertrages und einer Grundtendenz zur Publikumsgesellschaft ${ }^{153}$ der objektiven Auslegung zu folgen, erscheint zunächst scheinbar als valides Argument und als ein Gebot der Fairness. Andererseits berücksichtigt dies gerade mit Blick auf vertragliche Gerechtigkeitsvorstellungen und privatautonome Bindung nicht den Umstand, dass die wesentliche Argumentation der selbsterstandenen objektivierten Gesellschaftsidentität im Kapitalgesellschaftsrecht aufgrund einer schwer überschaubaren Gesellschaftermenge als bloße Geldgeber und Gewinnbeteiligungsberechtigte selbst für große Familiengesellschaften regelmäßig nicht greift. Die Familiengesellschaft ist vielmehr darauf angelegt, die hergebrachten Werte und das außerstatutarische Regelungsregime aufrechterhalten zu wollen, was jedenfalls im zurechenbaren Erkenntnisbereich der Gesellschafter Fortbestand haben soll.

Konsequenz einer gespaltenen Auslegung ist, dass außerstatutarische Vereinbarungen gegenüber solchen Gesellschaftern im Rahmen der Auslegung weiterhin berücksichtigt werden müssten. Gleichzeitig würde der mit der objektiven Auslegung bezweckte Schutz von Neugesellschaftern, denen die fehlende Kenntnis von der außerstatutarischen Vereinbarung nicht vorzuwerfen ist und die damit als schutzbedürftig erkannt werden, nicht vernachlässigt oder gar aufgehoben. Die gespaltene Auslegung schützt Vertrauen dort, wo es zurechenbar gesetzt und im Einzelfall enttäuscht worden ist. Propagierte man hingegen eine stets einheitliche Auslegung, könnten außerstatutarische Vereinbarungen allein durch die Aufnahme eines „gutgläubigen“ Neugesellschafters ihre Wirkung verlieren, obwohl sie von allen anderen Gesellschaftern bisher als verbindlich anerkannt worden sind. Soweit diese außerstatutarischen Vereinbarungen ${ }^{154}$ generell hinter ein verändertes Gesellschafterbild zurückträten, zerstörte dies gleichsam jegliches Vertrauen in begleitende Vereinbarungen, deren Aufnahme in den Gesellschaftsvertrag vielfach schon aus nichtjuristischen, etwa rein emotionalen Erwägungen bislang ausgeblieben ist. Eine

BGH, Urt. v. 20.1.1983 - II ZR 243/81, BB 1983, 996.

152 BGHZ 123, 347; BGHZ 116, 359; BGHZ 96, 245, 250; SchÄFER, aaO (Fn. 69), \ 2 GmbHG Rdn. 27; Fastrich, aaO (Fn. 69), \ 2 GmbHG Rdn. 31; Reuter, Münchener Komm. z. BGB, 7. Aufl., 2015, \25 Rdn. 23; Vetter, aaO (Fn. 70), \$23 AktG Rdn. 26.

153 BGH NZG 2013, 1334, Rdn. 20; Oetker, HGB, 4. Aufl., 2015, \ 161 Rdn. 138 m.w.N; Grunewald, ZGR 1995, 68, 71 ff; CoIng, ZGR 1978, 659.

154 Vgl. zur streitigen Rechtsnatur FLEISCHER, ZIP 2016, 1509. 
solche rechtliche Übergriffigkeit des Hinzutretens eines neuen Gesellschafters mit Blick auf das bisherige Gesellschaftsstatut ist für den Gesellschafterbestand quo ante weder erwartungsgemäß noch sinnvoll.

Entgegen dieser Ausführungen konstatiert die wohl h.M., dass körperschaftsrechtliche Satzungsbestimmungen zumindest dann rein objektiv und damit für alle Gesellschafter gleich auszulegen seien, wenn neue Gesellschafter hinzugekommen sind und diese von außerstatutarischen Umständen weder Kenntnis hatten noch Kenntnis haben mussten. ${ }^{155}$

Argumentativ wird gegen die gespaltene Auslegung eines (GmbH-)Gesellschaftsvertrages angeführt, dass sich dieser an eine unbestimmte Anzahl an Personen richte und daher eine „einheitliche, allgemein gültige und allein am urkundlichen Text orientierte Auslegung " zwingend sei. ${ }^{156}$ Weiterhin könnte bei Körperschaften generell erwogen werden, dass diese unabhängig von dem Bestand ihrer Mitglieder gedacht seien und daher auch ihre "Verfassung“ losgelöst von den Gesellschaftern und damit auch von allem außerhalb des Gesellschaftsvertrages Stehendem - stets objektiv - betrachtet werden müssten. ${ }^{157}$ Noch einen Schritt weiter ginge die Erwägung, dass der Gesellschaftsvertrag einer Korporation sich - gleichsam der Gesetzesidee - jedenfalls auch an die Allgemeinheit richte und daher einheitlich ausgelegt werden müsse. ${ }^{158}$ Dementsprechend leiten auch Ulmer und Löbbe die Notwendigkeit einer einheitlichen Auslegung aus der Rechtsnatur des Gesellschaftsvertrages als Organisationsstatut der Gesellschaft und dem zwingenden Bedürfnis der unterschiedslosen Maßgeblichkeit für gegenwärtige und künftige Gesellschafter $a b .{ }^{159} \mathrm{Zudem}$ sei die gespaltene Auslegung auch für die bisherigen Gesellschafter nur schwer zu ertragen, was jedoch bislang ohne Beleg oder Detailbegründung geblieben ist. ${ }^{160}$

Schließlich wird auf die Schwierigkeit einer präzisen Trennung der verschiedenen Auslegungen hingewiesen, so dass die subjektive Auslegung im Zweifel auch mittelbare Konsequenzen für Neugesellschafter mit sich bringen könnte. ${ }^{161}$ Ähnlich formuliert zunächst Wiedemann, der ebenfalls davon ausgeht, dass eine Unterscheidung nach dem Interesse neuer und alter Mitglieder nicht

155 Nachweise bei SchockenHoff, ZGR 2013, 76, 86.

156 BGHZ 14, 25, 36f; OLG Düsseldorf ZIP 1987, 227, 230; Sсноскеnhoff, ZGR 2013, 76, 88 m.w.N.

157 Vgl. Pfisterer, in: Saenger/Inhester, GmbHG, 3. Aufl., 2016, \2 Rdn. 11; Schäfer, aaO (Fn. 69), $\$ 2$ GmbHG Rdn. 27; Wiedemann, Sonderheft DNotZ 1977, 99, 105.

158 Scholz/Emmerich, Komm. z. GmbHG, 11. Aufl., 2012, \2 Rdn. 34 f.

159 Ulmer/Löbbe, in: Ulmer, Komm. z. GmbHG, 2. Aufl., 2013, 22 Rdn. 195; SCHOckenHOFF, ZGR 2013, 76, 88 m. w. N.

160 Ulmer/Löbbe, aaO (Fn. 159), $\$ 2$ GmbHG Rdn. 195.

161 Ulmer/Löbbe, aaO (Fn. 159), $\$ 2$ GmbHG Rdn. 195. 
möglich sei, da sämtliche Klauseln für den neuen Gesellschafter gleichermaßen Bedeutung gewinnen dürften wie für Altgesellschafter. ${ }^{162}$

In einem anderen Kontext - im Verhältnis zwischen Innen- und Außenverhältnis - hat sich Wiedemann aber für eine gespaltene Auslegung ausgesprochen. ${ }^{163}$ Lediglich für die Auslegung im Außenverhältnis, d.h. im Verhältnis zu den Gläubigern der $\mathrm{GmbH}$, müsse allein auf die objektive Auslegung abgestellt werden. ${ }^{164}$ Diese Unterscheidung, wonach eine gespaltene Auslegung zwar im Vergleich von Innen- zu Außenverhältnis, nicht aber auch im reinen Innenverhältnis zwischen schutzwürdigen und nicht schutzwürdigen Gesellschaftern möglich sein soll, ist schwer nachvollziehbar. Der Grundgedanke hinter der gespaltenen Auslegung ist die Schutzbedürftigkeit betroffener Mitgesellschafter wie auch der Gesellschaftsschutz gegen vertragsbrüchige Sozien. Wenn danach aber sowohl der Dritte im Außenverhältnis als auch der nicht an der außerstatutarischen Vereinbarung beteiligte Neugesellschafter durch eine objektivierte Auslegung geschützt werden, bestehen jedenfalls dann keine Unterschiede, wenn eine gespaltene Auslegung der fraglichen Klausel mit Blick auf Logik und Durchführung möglich ist. Nur wenn diese Option ausscheidet, verbleibt ein zwingender Grund für den Vorrang des Schutzes des Neugesellschafters.

Einer gespaltenen Auslegung kann darüber hinaus auch nicht entgegengehalten werden, dass es sich bei dem Grundsatz der Einheitlichkeit der Auslegung um einen „fundamentalen Grundsatz“ der Auslegung handelte, der aus dem Gleichheitsgebot, dem Gebot der Rechtssicherheit oder dem der Gewaltenteilung folge, da sämtliche dieser Grundsätze nur bei der Gesetzesauslegung, nicht aber auch bei der Auslegung privatrechtlicher Verträge und damit eines Gesellschaftsvertrages Bedeutung haben. ${ }^{165}$ Dementsprechend hält Schockenhoff mit einiger Berechtigung den vermeintlichen Grundsatz einer einheitlichen Auslegung für ein „Kryptoargument“, das den Blick auf die maßgeblichen Wertungen, wie insbesondere der Schutzbedürftigkeit der Einzelnen, verstelle. ${ }^{166} \mathrm{Im}$ Übrigen werden selbst Gesetze heutzutage zum Teil schon gespalten ausgelegt. Eine solche gespaltene Auslegung einer Norm findet sich beispielsweise dort, wo diese - zumindest für einzelne Personengruppen richtlinienkonform auszulegen sind. Es sei pars pro toto an den Umfang des Nacherfüllungsanspruchs im Kaufvertragsrecht erinnert. ${ }^{167}$ So wird für Verbraucher aufgrund der Verbrauchsgüterkaufrichtlinie angenommen, dass der

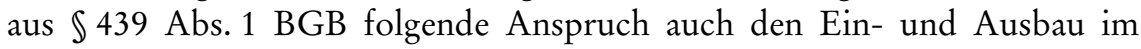

162 Wiedemann, Sonderheft DNotZ 1977, 99, $106 \mathrm{f}$.

163 Wiedemann, Sonderheft DNotZ 1977, 99, $107 \mathrm{f}$.

164 Wiedemann, Sonderheft DNotZ 1977, 99, $107 \mathrm{f}$.

165 SChOckenhoff, ZGR 2013, 76, 88.

166 Schockenhoff, ZGR 2013, 76, 88.

167 So auch Westermann, Münchener Komm. z. BGB, 7. Aufl., 2016, $\ 439$ Rdn. 15. 
Rahmen der Nacherfüllung umfasst, wohingegen ein solcher Anspruch für Unternehmer auf derselben normativen Basis stets verneint wird. ${ }^{168}$

Auch dürfte das überwiegend angenommene Gebot objektiver Auslegung im Kapitalgesellschaftsrecht keine tragfähige Argumentation gegen eine gespaltene Auslegung bieten. ${ }^{169}$ So hat schon das RG für den Verein entschieden, dass grundsätzlich auch die Entstehungsgeschichte des Gesellschaftsvertrages oder die „innerverbandliche Übung“ berücksichtigungsfähig sein sollen. ${ }^{170}$ Der BGH hat diesen Ansatz in der bereits angesprochenen Kerbnägel-Entscheidung aufgegriffen und fortgeführt. ${ }^{171}$

Im Übrigen lässt sich die Möglichkeit der gespaltenen Auslegung auch mit allgemein zivilrechtlichen Grundsätzen belegen. Die Frage nach einer gespaltenen Auslegung ist kein auf das Gesellschaftsrecht beschränktes Problem, sondern dem Zivilrecht bei Mehrpersonenverträgen immanent. Bei konsequenter Anwendung der $\mathbb{S} \mathbb{S} 133,157 \mathrm{BGB}$ ist es daher nicht nur denkbar, sondern häufig sogar tatsächlich der Fall, dass die an $\mathbb{S} \$ 133,157$ BGB ausgerichtete Auslegung zu divergierenden Empfängerhorizonten führt. ${ }^{172}$ In dem Fall, dass ein und dieselbe Erklärung, die an mehrere Personen adressiert ist, danach unterschiedlich verstanden werden durfte, kann diese nach der herrschenden Vertragsrechtsdoktrin jedenfalls dann unterschiedlich ausgelegt werden, wenn sich das begründete Rechtsverhältnis nach verschiedenen Personen aufteilen lässt. ${ }^{173}$ Erst wenn eine solche Aufteilung nicht mehr in Betracht kommt, soll für alle Beteiligten einheitlich die normative, d.h. objektive Auslegung gelten. ${ }^{174}$ Eine Übertragung dieser Grundsätze auf das Gesellschaftsrecht erscheint nicht zuletzt mit Blick auf das individuell gesetzte Vertrauen besonders in Familiengesellschaften sowohl erforderlich als auch sinnvoll. Anders als bei den Kritikern der gespaltenen Auslegung angeklungen, ist es nämlich gerade die Familiengesellschaft, die ihren erkennbaren begleitenden Umständen entsprechend kein Abstraktum vom Willen ihrer Gründer und ihrer gesellschaftsvertraglichen Umwelt bilden will. Die Einflechtung familiären Vertrauens und die Bindung aller, die zurechenbare Kenntnis hiervon haben oder haben müssen, wird ungeschriebener Vertragsbestandteil, was wohl auch der BGH so

168 Vgl. Berger, in: Jauernig, Komm. z. BGB, 16. Aufl., 2015, $\ 439$ Rdn. 20; WesterMANN, aaO (Fn. 167), \439 BGB Rdn. 15.

169 RG JW 1936, 2387; RGZ 159, 272, 278; Wiedemann, Sonderheft DNotZ 1977, 99, 105 m. w. N., 107; GRUNEWALD, ZGR 1995, 68, 86.

170 RG JW 1936, 2387; WiedemanN, Sonderheft DNotZ 1977, 99, 105 m.w. N.

171 BGH, Urt. v. 20.1.1983 - II ZR 243/81 = BB 1983, 996.

172 FleisCHER, DB 2013, 1466, 1472.

173 Fleischer, DB 2013, 1466, 1472 m.w. N.; Flume, BGB AT, Bd. II, 3. Aufl., 1979, S. 304.

174 Fleischer, DB 2013, 1466, 1472. 
sieht. ${ }^{175}$ Mit Blick auf die Argumentationslast müssen sich denn auch die Kritiker fragen lassen, weshalb die Enttäuschung des Vertrauens in ein bestimmtes Stimmverhalten mit Blick auf den Erhalt von Familienwerten etwa durch Reinvestition, Akzeptanz einer Buchwertklausel oder Nutzung des Familiennamens als Werbematerial allein deshalb den Schutz von Gesellschaftern und vertrauenden Sozien begründen sollte, weil lediglich ein redlicher Neugesellschafter in Unkenntnis hinzugetreten ist. Die Kritiker der gespaltenen Auslegung überspielen die materiellen Sorgen von Mitgesellschaftern und Gesellschaft zu Gunsten der rein formalen Behauptung, dass ein einheitliches Verständnis geboten sei. Die Familiengesellschaft ist ein leuchtendes Beispiel des Gegenteils. Soweit aber Altgesellschafter ihrerseits nicht schutzwürdig sein sollten, da etwa gegen diese im Rahmen eines Rechtsstreits dargelegt und bewiesen würde, dass eine Aufklärung oder Erörterung einer eingetretenen Rechtsunsicherheit hätte veranlasst werden müssen, um gegenüber den Mitgesellschaftern zumutbar vom Fortbestehen des gesetzten Vertrauens ausgehen zu dürfen, ist freilich auch eine Abweichung von der gespaltenen Auslegung denkbar. Diese Abweichung muss sich dann aber aus einem auch für die Altgesellschafter erkennbaren Umstand ergeben, der über das bloße Hinzutreten eines neuen Gesellschafters hinausgeht und von einem vernunftgeleiteten Empfängerhorizont aus zum Handeln veranlasst hätte. Der Schutz gegen solche Einwände, die leichtfertig erhoben werden könnten, ist abermals die Verteilung von Darlegungs- und Beweislast auf jeden, der sich hierauf beruft.

\section{Potentielle Drittbetroffenheit}

Wird einmal akzeptiert, dass Familiengesellschaftsstatuten ebenenübergreifend - also auch unter Heranziehung vertraglicher Abreden oder faktischer Verhältnisse außerhalb des Gesellschaftsvertrags - auszulegen sind, wenn die Auslegungsfrage zwischen Gesellschaftern streitig wird, so muss dies unabhängig davon gelten, ob die streitentscheidende statutarische Regelung potenziell auch Dritte betrifft. Wollte man dies anders sehen, bliebe letztlich kein Raum für eine subjektiv-ebenenübergreifende Auslegung, da sich praktisch zwischen jeder Regelung und den Interessen außenstehender Dritter ein indirekter, mittelbarer Zusammenhang konstruieren lassen dürfte. Beispielhaft sei die Ergebnisverwendung in der $\mathrm{GmbH}$ angeführt: Sofern diese unter strikter Beachtung bilanzieller Vorschriften, insbesondere des Vorsichtsprinzips $(\mathbb{} 252$ Abs. 1 Nr. 4 HGB) sowie der "Ausschüttungssperre“ des $\$ 30$ GmbHG erfolgt, sind Gläubiger hiervon akut und unmittelbar zweifelsohne nicht betroffen, da ihre Ansprüche de jure als werthaltig gelten. Potenziell und indirekt sind sie es aber gleichwohl, da es für die Bonität der Gesellschaft und das 
Ausfallrisiko der Gläubiger durchaus einen Unterschied bedeutet, ob das Jahresergebnis an die Gesellschafter ausgeschüttet oder aber - etwa aufgrund einer außerstatutarischen Abrede - zur Bildung einer eigenkapitalstärkenden Gewinnrücklage verwendet wird. Der Streit über die Ergebnisverwendung, in dem eine Gesellschafterminderheit ihre treuwidrige Aushungerung ${ }^{176}$ geltend macht und die Mehrheit dem entgegenhält, dass die statutarisch nicht vorgeschriebene Gewinnthesaurierung „jahrelang gelebter Praxis“ oder gar einer in der Familienverfassung niedergelegten Maxime der größtmöglichen „Stärkung der Eigenkapitalbasis des Familienunternehmens" folgt, ist demgemäß ohne Rücksicht auf Dritte - die letztlich sogar primäre Nutznießer der Thesaurierung sind $-\mathrm{zu}$ entscheiden.

Quintessenz dieser Überlegungen ist, dass nicht die potenzielle Betroffenheit Dritter durch den Gehalt einer statutarischen Regelung entscheidend ist, sondern allenfalls ihre Beteiligung an einer konkreten Auseinandersetzung über den Regelungsgehalt ${ }^{177}$, sofern die Auseinandersetzung keine geteilte Entscheidung erlaubt, was letztlich prozessual wie materiell-rechtlich am Maßstab der notwendigen Streitgenossenschaft i.S.v. $\$ 62 \mathrm{ZPO}$ zu messen wäre. Dementsprechend steht, um das vorherige Beispiel aufzugreifen, in einem Anfechtungsprozess analog $\int \mathbb{S} 241 \mathrm{ff}$. AktG, in dem die Fehlerhaftigkeit des Ergebnisverwendungsbeschlusses unter Berufung auf jene außerstatutarischen Gründe geltend gemacht wird, entgegen der herrschenden Meinung nicht schon die erga omnes-Wirkung des Urteils der Berücksichtigung außerstatutarischer Regelungsebenen entgegen. Dies entspricht im Ergebnis der KerbnägelRechtsprechung, beruht aber nicht auf prozessökonomischen Erwägungen, sondern auf der Wertung, dass der Auslegungstatbestand bei der Auslegung von Familiengesellschaftsstatuten nicht a priori, sondern allenfalls in begründeten Ausnahmefällen - nämlich bei akuter und unmittelbarer Betroffenheit Dritter - durch das Postulat objektiver Auslegung begrenzt sein darf. Prozessual bedeutete dies, dass nur im Fall der akuten Drittbetroffenheit hierfür auch eine notwendige Streitgenossenschaft i.S.d. $\$ 62$ ZPO bei einem solchen Anfechtungsfall in Betracht kommt. Diese Differenzierung wird jedoch in der höchstrichterlichen Rechtsprechung bislang nicht vorgenommen, ${ }^{178}$ so dass sich für den hier zuletzt vertretenen Ansatz keine höchstrichterliche Stütze findet. Allerdings wird die überschießende Wirkung der Berücksichtigung potenzieller Drittinteressen in einer Entscheidung des KGs aus dem Jahr $2015^{179}$ deutlich: Die Satzung einer familienbeherrschten $\mathrm{GmbH}$ enthielt eine

176 Vgl. etwa OLG Nürnberg v. 9.7.2008 - 12 U 690/07, DB 2008, 2415; Scholz/Verse, Komm. z. GmbHG, 11. Aufl., 2012, \29 Rdn. 54.

177 Vgl. in diese Richtung auch bereits SchockenHoff, ZGR 2013, 76, 88.

178 Dementsprechend stets für eine notwendige Beiladung BGHZ 122, 211, $240=$ NJW 1993, 1976; BGH NJW 1999, 1638, 1639.

179 KG v. 23.7.2015 - 23 U 18/15, GmbHR 2016, $29 \mathrm{ff}$. 
Öffnungsklausel zur Einrichtung eines Aufsichtsrats mit einfachem Mehrheitsbeschluss. Als sich Streit im Gesellschafterkreis anbahnte, richtete die (geschäftsführende) Gesellschaftermehrheit gegen die (ebenfalls geschäftsführende) Minderheit auf Grundlage der Mehrheitsklausel einen Aufsichtsrat ein, der als erste Amtshandlung den Minderheitsgesellschafter-Geschäftsführer abberief. Das KG bewertete die Abberufung als unwirksam, da schon die Einrichtung des Aufsichtsrats nicht wirksam erfolgt sei. Denn es handele sich um eine dauerhafte Änderung der Gesellschaftsverfassung - was im Falle eines echten, mit Überwachungs- und insbesondere Personalkompetenz ausgestatteten Aufsichtsrat zweifelsohne zutrifft -, die nicht durch eine statutarische Öffnungsklausel, sondern ausschließlich unter Beachtung der zwingenden Bestimmungen der $\int \mathbb{5} 53,54 \mathrm{GmbHG}$ ins Werk gesetzt werden könne ${ }^{180}$. Begründet wird die Entscheidung damit, die zwingenden $\$ \mathbb{S} 53,54 \mathrm{GmbHG}$ könnten nicht durch "gesellschaftsvertragliche Abmachungen (,Öffnungsklauseln") außer Kraft gesetzt werden ", selbst wenn das qualifizierte Mehrheitserfordernis des $\$ 53$ Abs. $2 \mathrm{GmbHG}$ sich möglicherweise dadurch überwinden lasse, dass die Öffnungsklausel als ,antizipierte Zustimmung aller, auch später binzutretender Gesellschafter " verstanden werde ${ }^{181}$; denn jedenfalls die übrigen Elemente der

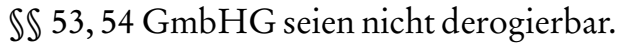

Dem ist zum einen - eher formalistisch - entgegenzuhalten, dass die $\mathbb{S} 53,54$ GmbHG bei Schaffung der Öffnungsklausel durchaus eingehalten wurden. Zum anderen stellt sich aber wertungsmäßig die Frage: cui bono? Der Rechtsverkehr kennt die Öffnungsklausel und ersieht aus der Einreichung einer Liste der Aufsichtsratsmitglieder gemäß $₫ 52$ Abs. 3 Satz 2 GmbHG, dass von ihr Gebrauch gemacht worden ist ${ }^{182}$. Für die Gesellschafter gilt dies erst recht. Bedenken hat das KG offenbar deshalb, weil die Satzung den Status „GmbH mit Aufsichtsrat" lediglich als Eventualität, nicht aber als Faktum - das erst durch den außerstatutarischen Umstand eines zusätzlichen Gesellschafterbeschlusses herbeigeführt wird - reflektiert. Letztlich bedeutet die Entscheidung des KGs, dass der konkrete und unter sogar den Anforderungen der $\mathbb{S} 53,54 \mathrm{GmbHG}$ entsprechend hinreichend präzise niedergelegte Wille der Gesellschafter(-mehrheit) aufgrund des abstrakten drittschützenden Charakters der $\$ \$ 53,54 \mathrm{GmbHG}$ ignoriert wird.

Letztlich steht mithin potentielle Drittbetroffenheit der Einbeziehung vertragsexterner Umstände in die Auslegung der Vertragsurkunde nicht entgegen. 


\section{Zusammenfassung in Thesenform}

1. Trotz erheblicher definitorischer Schwierigkeiten bei der Erfassung der „Familiengesellschaft“ bringt diese je nach Ausprägung gesellschaftsvertraglich relevante Besonderheiten mit sich, deren Beachtung mit Blick auf die Vertragsfreiheit der Gesellschafter beachtlich ist.

2. Zentraler Anknüpfungspunkt der rechtlichen Berücksichtigung und Einflechtung dieser Besonderheiten ist die Auslegung des Gesellschaftsvertrages, der hierdurch eine überragende Bedeutung zukommt.

3. Die Auslegung des Gesellschaftsvertrages überwindet die Mehrschichtigkeit des Familienunternehmens, das regelmäßig neben dem Gesellschaftsstatut weitere Abreden, besondere Begleitumstände, spezifische Vertragsverständnisse und sogar ganze Regelwerke in Form einer Familienverfassung aufweist.

4. Andere zu Gebote stehende Kontrollmechanismen wie die Rahmenordnung der $\int \mathbb{S} 134,138$ Abs. 1 BGB, inhaltsorientierte Klauselkontrollen in Anlehnung an das AGB-Recht nach $\mathbb{2} 242$ BGB sowie die gesellschaftsrechtliche Treuepflicht sind ihrem Gegenstand nach strikt an das Ergebnis einer sorgsamen Auslegung gebunden, so dass einer Kontrolle nur das Auslegungsergebnis, nicht der Auslegungsvorgang oder gar die Auslegungsgrundlage unterworfen wäre.

5. Veränderungen in der Gesellschafterstruktur seit Gründung werfen die Frage nach dem Schutz eines redlichen Neugesellschafters ebenso wie nach der Wahrung des Vertrauens der Bestandsgesellschafter auf. Die Abwägung der widerstreitenden Interessen führt zu einer weitreichenden Zurechenbarkeit nutzbarer Erkenntnisquellen durch den Neugesellschafter, deren einhergehende rechtliche Schärfe durch die prozessuale Verteilung von Darlegungs- und Beweislast angeglichen wird.

6. Entgegen der bislang wohl h.M. in Literatur und Rechtsprechung ist im Fall schutzwürdiger Neugesellschafter eine gespaltene Auslegung des Gesellschaftsvertrages zu befürworten, wonach dessen Auslegung ein anderes Ergebnis zeitigt, je nach Betroffenen- und Betrachterwinkel. Das materielle Gebot des Schutzes bedeutsamer Vertrauensinteressen überwiegt dabei regelmäßig den Einwand denkbarer Rechtsunsicherheiten durch Aberrationen.

7. Die Feststellungen zu ebenübergreifender Auslegung und gespaltenem Auslegungsverständnis müssen in der Konsequenz auch dann gelten, wenn Dritte hiervon potentiell betroffen sind. Die Grenze ist erst dort erreicht, wo Dritte tatsächlich im Rahmen einer rechtlichen Auseinandersetzung ein konkretes Interesse an der sie schützenden Auslegung geltend machen und im Rahmen dieser Auseinandersetzung eine von Rechts wegen zwingende Einbeziehung der Mit(Alt-)gesellschafter zu erfolgen hat. 